\title{
Combinatorial effects on gene expression at the Lbx1/Fgf8 locus resolve Split-Hand/Foot Malformation type 3
}

Giulia Cova ${ }^{1,2,13}$, Juliane Glaser ${ }^{1}$, Robert Schöpflin ${ }^{1,2,3}$, Salaheddine Ali ${ }^{1,2}$, Cesar Augusto PradaMedina $^{1,14}$, Martin Franke ${ }^{1,2,15}$, Rita Falcone ${ }^{1}$, Miriam Federer ${ }^{1,16}$, Emanuela Ponzi ${ }^{4}$, Romina Ficarella ${ }^{4}$, Francesca Novara ${ }^{5}$, Lars Wittler ${ }^{6}$, Bernd Timmermann?, Mattia Gentile ${ }^{4}$, Orsetta Zuffardi $^{8}$, Malte Spielmann ${ }^{9,10,11}$, Stefan Mundlos ${ }^{1,2,12, *}$

${ }^{1}$ Max Planck Institute for Molecular Genetics, RG Development \& Disease, Berlin, 14195, Germany

${ }^{2}$ Institute of Medical and Human Genetics, Charité Universitätsmedizin Berlin, Berlin, 10117, Germany

${ }^{3}$ Department of Computational Molecular Biology, Max Planck Institute for Molecular Genetics, Berlin, 14195, Germany

${ }^{4}$ Medical Genetics Unit, Department of Reproductive Medicine, ASL Bari, Bari, 70131, Italy

${ }^{5}$ Microgenomics Laboratory, Pavia, 27100, Italy

${ }^{6}$ Department of Developmental Genetics, Transgenic Unit, Max Planck Institute for Molecular Genetics, Berlin, 14195, Germany

${ }^{7}$ Sequencing Core Facility, Max Planck Institute for Molecular Genetics, Berlin, 14195, Germany ${ }^{8}$ Department of Molecular Medicine, University of Pavia, Pavia, 27100, Italy

${ }^{9}$ Institute of Human Genetics, Universitätsklinikum Schleswig Holstein Campus Kiel and ChristianAlbrechts-Universität, Kiel, 24118, Germany 
${ }^{10}$ Institute of Human Genetics, University of Lübeck, Lübeck, Germany

${ }^{11}$ Human Molecular Genomics Group, Max Planck Institute for Molecular Genetics, Berlin, 14195,

Germany

${ }^{12}$ Berlin-Brandenburg Center for Regenerative Therapies (BCRT), Charité Universitätsmedizin

Berlin, Berlin, 13353, Germany

${ }^{13}$ Present address: Department of Pathology, New York University School of Medicine, Langone

Health Medical Center, New York, NY, 10016, USA

${ }^{14}$ Present address: Kennedy Institute of Rheumatology, University of Oxford, Oxford, OX3 7FY, UK

${ }^{15}$ Present address: Centro Andaluz de Biología del Desarrollo, Consejo Superior de Investigaciones Científicas/Universidad Pablo de Olavide, Seville, 41013, Spain

${ }^{16}$ Present address: Universität Innsbruck, Innsbruck, 6020, Austria

*Corresponding author: mundlos@molgen.mpg.de 


\begin{abstract}
Split-Hand/Foot Malformation type 3 (SHFM3) is a congenital limb malformation associated with tandem duplications at the LBX1/FGF8 locus. Yet, the disease patho-mechanism remains unsolved. Here we investigated the functional consequences of SHFM3-associated rearrangements on chromatin conformation and gene expression in vivo in transgenic mice. We show that the Lbx1/Fgf8 locus consists of two separate, but interacting, regulatory domains. Re-engineering of a SHFM3-associated duplication and a newly reported inversion in mice resulted in restructuring of the chromatin architecture. This led to an ectopic activation of the Lbx1 and Btrc genes in the apical ectodermal ridge (AER) in an Fgf8-like pattern. Artificial repositioning of the AER-specific enhancers of Fgf8 was sufficient to induce misexpression of Lbx1 and Btrc. We provide evidence that the SHFM3 phenotype is the result of a combinatorial effect on gene misexpression and dosage in the developing limb. Our results reveal new insights into the molecular mechanism underlying SHFM3 and provide novel conceptual framework for how genomic rearrangements can cause gene misexpression and disease.
\end{abstract}




\section{Introduction}

At the sub-megabase scale, chromosomes are organized into distinct regions of high interaction called Topologically Associating Domains (TADs) that are separated from each other by boundaries $^{1,2}$. TADs are thought to restrict enhancer-promoter contacts thereby defining regulatory domains. Structural variations (SVs), such as deletions, duplications and inversions, can disrupt these functional units and different types of SVs can induce distinct topological changes. For instance, deletions are mainly responsible for TAD fusion, duplications can promote formation of a new TAD (neo-TAD), while TAD shuffling is frequently observed upon an inversion $^{3-5}$. SVs can not only affect gene dosage but also modulate basic mechanisms of gene regulation. Indeed, SVs can alter the copy number of regulatory elements or modify the 3D genome by disrupting higher-order chromatin organisation. This can give rise to ectopic enhancer-promoter contacts, gene misregulation and ultimately disease ${ }^{5}$. However, the general applicability of this concept remains under investigation. Studies in Drosophila, for example, demonstrated that the disruption of TADs and enhancer-promoter interactions are not necessarily accompanied by changes in expression, indicating a high degree of robustness of the genome against such events ${ }^{6}$.

SHFM is a congenital limb malformation characterized by variable defects of the central rays of the autopod often together with syndactyly and/or aplasia/hypoplasia of the phalanges, metacarpals, and metatarsals ${ }^{7}$. SHFM is known as a paradigm for genetic heterogeneity. While duplications on either $17 \mathrm{p} 13.3$ or $10 \mathrm{q} 24^{8}$ are the most frequent genetic cause, SHFM can be also associated with coding and non-coding mutations at other loci. Indeed, SVs involving the DYNC1/1 locus where regulatory elements for $D L X 5$ and $D L X 6$ are localized ${ }^{9}$, and mutations in TP63, $D L X 5$, 
or $W N T 10 B^{10}$ have been previously associated with SHFM. The common underlying pathomechanism of these mutations is thought to be the failure to maintain a proliferation/growth signal from the apical ectodermal ridge (AER), a signalling centre of ectodermal cells at the distal end of the limb bud ${ }^{11}$. SHFM is also a clinically heterogeneous disease ${ }^{12}$, as some patients carrying SHFM-associated SVs do not show any limb phenotype or a very mild one, suggesting that SHFM patho-mechanism is not merely associated to gene dosage alteration.

Tandem duplications on chromosome $10 q 24$ at the LBX1/FGF8 locus are considered the cause for SHFM type $3(\text { SHFM3 })^{13}$, but the specific patho-mechanism of SHFM3 associated duplications remains unsolved. The reported duplications are at least $500 \mathrm{~kb}$ in size, encompassing five genes of the locus ( $L B X 1, B T R C, P O L L, D P C D$, and FBXW4) but excluding the neighbouring FGF8 (Supplementary Fig. 1) $)^{14}$. While $L B X 1$ is essential for early muscle cell differentiation and migration $^{15}$, FGF8 is expressed in the AER together with other FGFs where it functions as a growth factor for the underlying mesechyme ${ }^{16} . B T R C$ is important in regulating cell cycle checkpoints ${ }^{17}$, $D P C D$ in the generation and maintenance of ciliated cells ${ }^{18}, P O L L$ encodes for a DNA polymerase ${ }^{19}$, whereas FBXW4 is involved in ubiquitin mediated protein degradation ${ }^{20}$. Duplications can have different effects on gene regulation depending on their relative position to chromatin domain boundaries. Studies of the SOX9 locus, for example, have shown that duplications within the SOX9 TAD can lead to SOX9 mis/overexpression and sex reversal, whereas duplications that include the SOX9/KCNJ2 boundary and the $K C N J 2$ gene result in $K C N J 2$ misexpression and brachydactyly ${ }^{4}$. In the latter situation, a neo-TAD is formed leading to contact of the SOX9 enhancers with the KCNJ2 promoter and KCNJ2 expression in a SOX9-like pattern. 
Given these examples, it is conceivable that the SHFM3-associated duplications may have specific regulatory effects that could result in gene misexpression.

In addition to the SHFM3 duplications at the LBX1/FGF8 locus, we reported here for the first time a new case of SHFM3 malformation associated with an inversion at the same locus. This inversion was also of particular interest as the patient is a good example of clinical heterogeneity of this limb malformation within the same individual (Supplementary Fig. 2). In order to uncover the molecular mechanism underlying this congenital disease, we engineered a duplication and the inversion at the $L b \times 1 / F g f 8$ locus in mice using CRISPR/Cas9 genome editing tool ${ }^{21}$. Chromosome conformation capture $\mathrm{Hi}-\mathrm{C}(\mathrm{cHi}-\mathrm{C})$ analysis of the mouse developing limbs in mutants showed that both SVs led to a disruption of the wild-type three-dimensional chromatin configuration of the locus and ectopic enhancer-promoter contacts. Strikingly, this caused misexpression of two genes, Lbx1 and Btrc, in an Fgf8-like pattern in the AER which appeared to be associated with a dose-dependent phenotype. Collectively, our results illustrate the impact of SVs at the Lbx1/Fgf8 locus on chromatin architecture and gene regulation and reveal the complex molecular pathomechanism underlying SHFM3 limb malformation. With the emergence of novel sequencing technologies and the improvement in the discovery and characterization of SVs, this study illustrates the myriad ways by which SVs can cause disease (i.e., a combination of enhancer repositioning and dosage effect), highlighting the challenging task of their interpretation in the clinic. 


\section{Results}

SHFM3-associated SVs include the Lbx1/Fgf8 domain boundary and the Fgf8 AER enhancers

To characterize the 3D conformation at the Lbx1/Fgf8 locus during mouse development, we performed cHi-C in wild-type murine limb buds at E11.5, a developmental stage where both $L b x 1$ and Fgf8 limb developmental genes are expressed (Fig. 1a). We observed that the locus is characterized by the presence of two main chromatin regulatory domains (Fig. 1a, indicated by black dashed lines), a centromeric and a telomeric containing $L b x 1 / B \operatorname{trc}$ and $F b x w 4 / F g f 8$ genes, respectively, and hereafter referred to as $L b x 1$ and Fgf8 TADs. These domains are separated from neighbouring TADs and from each other by CTCF-associated TAD boundaries in the classical divergent orientation ${ }^{22}$ (Fig. 1a). Poll and $D p c d$ are located within the boundary region separating Lbx1 and Fgf8 TADs. Given the divergent roles and expression pattern of $L b x 1$ and Fgf8 during muscle and limb development, respectively ${ }^{23,24}$, separated regulatory domains can be expected to ensure correct gene regulation. The Fgf8 TAD contains several well characterized enhancers with AER-specific activity as well as other tissue-specific enhancers corresponding to the Fgf8 expression pattern in the embryo, such as the branchial arches, the somites and the midbrainhindbrain boundary ${ }^{25,26}$. Interestingly, all identified AER enhancers $(58,59,61,66$; yellow ovals in Fig. 1a) are located within a $40 \mathrm{~kb}$ region in the introns of Fbxw4, with the exception of one enhancer (80; orange oval in Fig. 1a) which is located in close proximity to Fgf8.

Virtual $4 C$ analysis with viewpoints on $L b x 1$ and Fgf8 further emphasized how the interactions of these genes are mainly restricted within their respective chromatin regulatory domains (interactions are highlighted in yellow in Fig. 1a). As Fgf8 and $L b \times 1$ are located very close to the centromeric and telomeric boundaries, respectively, we could appreciate the interactions with 
the TAD boundary region (highlighted in lilac in Fig. 1a) defining the two domains. Despite being two separated domains, the Lbx1 and Fgf8 TADs are also connected via an additional loop (Fig. 1a, top dashed circle in cHi-C map). This interaction across the boundary is also clearly visible in our 4C analysis (highlighted in pink in Fig. 1a) indicating that subpopulations of limb bud cells can have different configurations, but also that a certain degree of leakiness of the Lbx1/Fgf8 boundary exists, leading to a certain level of intermingling of the two domains in spite of the very different expression patterns of $L b x 1$ and Fgf8.

Mapping of in-house and already published SHFM3 duplications onto the 3D structure of the locus showed that they all include the boundary between the Lbx1 and Fgf8 TADs (Fig. 1b). Furthermore, all duplications comprised $L b x 1$ at the centromeric side and the previously identified Fgf8 AER enhancers located within Fbxw $4^{26}$, while excluding Fgf8 at the telomeric side

(Fig. 1b and Supplementary Fig. 1). Upon mapping of our new case of SHFM inversion we observed that the boundary region between the $L b x 1$ and Fgf8 TADs and the Fgf8 AER enhancers are involved also in this SHFM-associated SV, suggesting a potential common patho-mechanism.

\section{SHFM3-associated duplication and inversion result in ectopic interactions of Fgf8 AER enhancers region with $L b \times 1$ TAD}

To investigate the effect of the SHFM3-associated duplications and inversion on chromatin configuration, gene expression and phenotype, we engineered a human SHFM3 duplication (Dup) and the newly reported inversion (Inv1) in mice (Fig. 1c). cHi-C was performed in homozygous Dup and Inv1 E11.5 murine limb buds (Fig. 2). The Dup cHi-C map (Fig. 2b) showed a general increase in the frequency of interactions within the duplicated DNA region compared to the wild- 
type (Fig. 2a), reflecting the copy number increase. Interestingly, in the KR-normalized subtraction map, in which the bias of the higher copy number is overcome (Fig. 2c), we observed an architectural stripe of increased interactions (indicated by black arrowheads in Fig. 2c) between part of the Fgf8 TAD containing the Fgf8 AER enhancers involved in the duplication and a region immediately upstream of the $L b x 1$ centromeric TAD boundary. This reflected the formation of a neo-TAD, as illustrated in the schematic (Fig $\mathbf{2 d}$ ). The neo-TAD, indicated by the blue/green triangle between the two Lbx1 TADs (yellow), contained only Fbxw4, the Fgf8 AER enhancers and a small ( $38 \mathrm{~kb})$ region flanking the centromeric side the $L b \times 1$ TAD boundary, thus unlikely to have a pathological effect by itself. However, we also observed increased contacts between the entire $L b \times 1$ TAD and the Fgf8 AER enhancers region (indicated by dashed rectangle and asterisks in Fig. $\mathbf{2 b}$ and $\mathbf{c})$. These interactions were likely established by the Fgf8 AER enhancers from the neo-TAD, with both the endogenous and duplicated Lbx1 TADs (red arrows in Fig. 2d). Indeed, in the Dup mutant, interaction between the Fgf8 AER enhancers and the 3' $L b \times 1$ TAD is favoured by a shorter distance (the $L b \times 1$ gene is located $\sim 130 \mathrm{~kb}$ in Dup versus $\sim 380 \mathrm{~kb}$ in wild-type configuration) and by the presence of a weaker TAD boundary (two CTCF binding sites instead of four). Moreover, the Fgf8 AER enhancers could also contact the 5' Lbx1 TAD due to the previously mentioned leakiness of the boundary and the absence of their target gene Fgf8, making these enhancers free to interact with the neighbouring $L b x 1$ gene and the entire $L b x 1$ TAD.

We observed ectopic interactions between the Fgf8 AER enhancers region and the $L b \times 1$ TAD also in Inv1 $\mathrm{cHi}-\mathrm{C}$ (Fig. $2 \mathrm{e}$ and $\mathbf{f}$ ). In the inversion, the boundary between Lbx1 and Fgf8 TADs functioned as an anchor point around which part of the Fgf8 TAD was moved into the Lbx1 TAD 
and vice versa. Due to the fact that the centromeric breakpoint of the inversion was relatively close to the boundary, the Lbx1 TAD was enlarged while the Fgf8 TAD was reduced in size. As a consequence, the Fgf8 AER enhancers were now relocated within this bigger Lbx1 TAD which exhibited increased contacts (dashed rectangle and asterisks in Fig. $2 \mathbf{e}$ and $\mathbf{f}$ ) with the repositioned Fgf8 AER enhancer region, underlining a common trait with the Dup. Additionally, upon such reshuffling, Fgf8 was now isolated from its own AER enhancers and we observed indeed loss of interactions between these elements and their endogenous gene (dashed rectangle with white asterisks in Fig. 2f).

Given that the overall configuration of the locus with two main TADs was conserved in humans

(Fig. 3a), we then investigated whether the observed interaction changes were also present in SHFM3 patients. We performed 4C-seq experiments in human fibroblasts from two patients carrying the SHFM3 duplication (fibroblasts from the inversion case were not available) and exhibiting the classical SHFM phenotype. Using viewpoints on FGF8 and on the two genes in the LBX1 TAD ( $L B X 1$ and $B T R C$ ), we detected ectopic interactions involving the FGF8 AER enhancers region and both $L B X 1$ and $B T R C$ which were not present in the healthy control (Fig. 3b). Comparison with mouse virtual $4 \mathrm{C}$ plots generated from Dup $\mathrm{cHi}-\mathrm{C}$ maps further confirmed that the same ectopic interaction existed also in our Dup mutants (Fig. 3c), revealing we generated here a genetic mutant mouse model for SHFM3.

\section{Dup and Inv1 result in ectopic expression of $L b \times 1$ and Btrc in an Fgf8-like pattern in the AER}

To investigate the effect of the observed chromatin rearrangements on gene expression, we performed detailed expression analysis (RNA-seq, single cell RNA-seq and whole mount in situ 
hybridisation (WISH)) at developmental stage E11.5. In the Dup mutant, all genes within the duplication (Lbx1, Btrc, Poll, Dpcd and Fbxw4) were significantly upregulated in the developing limb buds, corresponding to the copy number increase (Fig. 4a). In the Inv1 mutant, Fbxw4 and Fgf8 were downregulated, reflecting the disruption of the gene and the repositioning of the Fgf8 AER enhancers, respectively (Fig. 4a). Strikingly, $L b x 1$ and Btrc exhibited increased expression levels in the Inv1 mutant, indicating that the observed chromatin rearrangement had an effect on gene expression. Genes outside of the Fgf8 and Lbx1 TADs (Sif2, Twnk and Oga) remained unchanged in both Dup and Inv1 mutants (Fig. 4a).

To investigate whether these changes of gene expression were specific to some regions of the limb bud, we performed single-cell RNA-seq (scRNA-seq) from E11.5 wild-type and Dup limb buds. Bioinformatic analysis revealed individual clusters corresponding to the major cell types in the developing limb including mesenchyme, dorso-ventral ectoderm, AER and muscle as well as satellite cell types such as neurons, lymphocytes, keratinocytes and endothelial cells (Fig. 4b). In wild-type cells, Fgf8 expression was only present in the AER, whereas $L b x 1$ was expressed only in muscle cells and the four other genes at the locus were expressed in all major cell types of the developing limb (Fig. 4c). The bulk RNA-seq data were confirmed such that the five genes included in the duplication (Lbx1, Btrc, Poll, Dpcd and Fbxw4), but not Fgf8, displayed increased expression in the Dup mutant compared to wt (Fig. 4c). Interestingly, $L b x 1$, and to a lesser extent Btrc, showed a strong AER expression in the Dup mutant, indicating misexpression in the AER on top of the increased copy number. This suggested that both $L b x 1$ and Btrc were activated by the Fgf8 AER enhancers now able to contact the two genes' promoters. Thus, the scRNA-seq data 
confirmed the bulk RNA-seq data and showed, in addition, that $L b x 1$ and Btrc were misexpressed in the AER.

Next, we performed WISH to confirm and further investigate potentially altered expression patterns. We uncovered that $L b x 1$, normally expressed in migrating muscle cells (black arrowheads for $L b x 1$ in Fig. 5a) and Btrc, a ubiquitously expressed gene, were ectopically expressed in the AER, resembling the Fgf8 specific expression pattern (Fig. 5a). As observed in the scRNA-seq data, $L b x 1$ misexpression appeared to be stronger than Btrc (Fig. 4c and Fig. 5a). Importantly, an even more pronounced misexpression of both genes was also observed in the Inv mutant (Fig. 5a). In the Dup mutant, Fgf8 expression was unchanged compared to wild-type. In contrast, in the Inv1 mutant we observed a loss of Fgf8 expression likely due to the loss of the endogenous interaction of Fgf8 with its enhancers (Fig. 5a). Finally, we showed that misexpression in the AER was not detected for Poll, Dpcd and Fbxw4, neither in Dup, nor in the Inv1 mutants (Supplementary Fig. 3a).

Next, we went on testing whether the ectopic expression of Btrc and $L b x 1$ in the AER of the Dup and Inv1 embryos was solely due to repositioning of the Fgf8 AER enhancers. To do so, we used CRISPR/Cas9 technology to insert four published essential Fgf8 AER enhancers (CE58, CE59, CE61, CE66) $)^{25,26}$ in the Lbx1 TAD in a wild-type background (Fgf8 AER enh KI, Fig. 5a). Given the presence of a CTCF binding site within enhancer CE58 and our aim to not create any new boundary upon knock-in we removed the CTCF recognition motif from enhancer CE58. WISH on Fgf8 AERenhancer KI E11.5 mutant embryos showed misexpression of Lbx1 and Btrc in the AER (Fig. 5a), supporting the ability of the Fgf8 enhancers to activate other genes. Of note, we observed that Lbx1 was ectopically expressed in the AER of all analysed embryos, while Btrc was present in the 
AER of $50 \%$ of the examined embryos and displayed a much lower signal. This result further confirmed that the observed ectopic expression of $L b x 1$ and Btrc in the AER was due to activation by Fgf8 enhancers.

\section{Lbx1 expression in the AER induces myogenic activation}

We and others have previously shown that ectopic gene expression during organ formation can lead to developmental malformation ${ }^{3,4,27-29}$. To investigate the molecular consequences of the high expression of $L b x 1$ in the developing $A E R$, we calculated the effect of the duplication on gene expression exclusively in the AER population, using the scRNA-seq data. We reasoned that $L b x 1$ could activate its gene regulatory network in the AER and to investigate this we performed a Gene Ontology (GO) search using the 200 genes most differentially up-regulated in the AER of the Dup mutant compared to wild-type (Supplementary Table 1). Strikingly, using the MGI mammalian phenotype terms, 4 out of the 5 most enriched terms were related to myogenic phenotypes (Fig. 5b). Genes associated with these terms included Lbx1, Myog, Gab1, Rela, Mapk14 and Dnm2 which were all expressed in an increased number of cells in the AER of the Dup and at a higher level compared to wild-type (Fig. 5c). Of note, $L b x 1$ expression was detectable in almost all AER cells while none of the wild-type AER cells expressed $L b \times 1$, corroborating our previous observations. This suggested that $L b x 1$ ectopic expression in the AER of the Dup mutant induced genes of the myogenic pathway. Additionally, expression of one of the genes, Myog, was also significantly increased in the bulk RNA-seq differential analysis data from limb bud in Inv1 mutant (Supplementary Table 2). 


\section{Inv1 but not Dup results in a SHFM-like phenotype}

Next, we analysed the mutant mice for skeletal phenotypes at developmental stage E18.5. In the Dup mutant we did not observe any skeletal malformations in the extremities of all the analysed transgenic embryos $(n=5)$ nor in $>25$ newborns and adult mice of the established Dup mouse line. In contrast, 3 out of 11 E18.5 Inv1 mutant embryos exhibited skeletal defects with a tendency to digit separation and partially missing or fused digits/bones (Fig. 5d), resembling the phenotype of SHFM patients ${ }^{13}$ and particularly that of the SHFM3 inversion individual (Supplementary Fig. 2). Indeed, clinical variability has been observed within SHFM families, between different families and also between limbs of a single individual ${ }^{13,30}$. Additionally, in $\ln v 1$ we found that forelimbs and hindlimbs were underdeveloped (Fig. 5e), a phenotype also observed in the Fgf8 conditional knock-out ${ }^{24,31}$. Loss of $F g f 8$ expression is known to affect the proper development of humerus, radius, ulna, and to cause loss mainly of the first digit ${ }^{24}$, a phenotype different from SHFM.

To further investigate the contribution of $L b x 1$ and Btrc to SHFM, we engineered two more CRISPR/Cas9 alleles to promote $L b x 1$ or Btrc misexpression alone in the AER and compared it to Dup and Inv1 where genes were simultaneously misexpressed. First, we generated a second, slightly larger inversion (Inv2) including the Fgf8 AER enhancers and Btrc (Supplementary Fig. 4b). This mutant showed ectopic interactions between the Fgf8 AER enhancers and Lbx1 only, as observed in $\mathrm{cHi}-\mathrm{C}$ and particularly in the subtraction $\mathrm{cHi}-\mathrm{C}$ map (Supplementary Fig. $4 \mathrm{c}$ and $4 \mathrm{~d}$ ) but in general with much lower intensity compared to Inv1 mutant. Misexpression of only $L b x 1$ but not Btrc was indeed detected in the AER (Supplementary Fig. 4a). To investigate the effect of an ectopic expression of Btrc alone, we re-targeted the Inv1 allele and deleted Lbx1 (Inv1 $\Delta L b \times 1)$ (Supplementary Fig. 4b). As expected, no misexpression for $L b \times 1$ was detected in the AER 
and in the limb in general, while Btrc was transcriptionally active (Supplementary Fig. 4a). In addition, we observed a weak expression of Fgf8 rescued in the AER, underlining the ability of the Fgf8 AER enhancers to re-contact their endogenous target gene in the absence of $L b \times 1$ and, as it happened in the Dup mutant, to overcome the boundary. Skeletal analysis of Inv2 and Inv1 $\Delta L b \times 1$ mutants (Supplementary Fig. 4f) revealed underdeveloped limbs due to a complete (Inv2) or strong (Inv1 $\Delta L b \times 1$ ) loss of Fgf8 expression, but none of them ( $\mathrm{n}=10$ for $\ln v 2$ and $\mathrm{n}=7$ for $\ln v 1$ $\Delta L b \times 1)$ showed the level of SHFM phenotype observed in Inv1, supporting the idea that misexpression of both $L b x 1$ and Btrc is required to develop a SHFM-like phenotype. Comparison between $\ln v 1$, Inv2 and $\ln v 1 \Delta L b \times 1$ further brought up a potential effect of the partial or complete loss of Fgf8 expression in triggering the onset of the SHFM-like phenotype in combination with Lbx1 and Btrc misexpression. Inv2 phenotype, where Fgf8 expression was completely lost, seemed indeed more severe than Inv1 $\Delta L b x 1$, where Fgf8 was only partially lost (Fig. 5d, Supplementary Fig. 4a, 4f and Fig. 6).

Finally, the level of misexpression of $L b \times 1$ and Btrc played probably also an important role in developing or not the limb malformation. This could be also particularly true for the Dup and Fgf8 AER-enhancer $K I$ mutants, where no striking phenotype was observed. Despite simultaneous misexpression of both $L b x 1$ and Btrc genes in the AER, the level of Btrc misexpression was lower than in the Inv1 mutant (Fig. 5a and 5d). 


\section{Discussion}

Split-hand/foot malformation (SHFM) is a congenital limb malformation affecting the central rays of the hands and/or feet (OMIM \#183600, \#313350, \#600095, \#605289, and \#606708). The condition is a classic example of allelic heterogeneity, phenotypic variability, and pleiotropy ${ }^{32}$. cHi-C of the Lbx1/Fgf8 locus, involved in SVs associated with SHFM type 3, showed that the region consists of two major TADs, each harbouring an important developmental gene with strikingly different expression patterns (Fig. 6). Fgf8 is contained in the Fgf8 TAD together with a number of well characterized enhancers that regulate Fgf8 specific expression in the limb AER and other tissues $^{25}$. $L b x 1$, a muscle-specific transcription factor expressed only in migrating muscle cells, is for its part comprised in the $L b x 1$ TAD. As expected, the two regulatory TADs are separated by a boundary with divergent CTCF sites. A combination of $\mathrm{cHi}-\mathrm{C}$ and $4 \mathrm{C}$ analysis demonstrated that the two domains are connected via a larger loop, established between the centromeric $L b \times 1$ TAD boundary and the telomeric Fgf8 TAD boundary, within which a certain degree of contact is maintained (Fig. 1a and Fig. 3). This complex arrangement was disrupted by the SHFM3 duplication (Dup) resulting in changes in the 3D chromatin architecture. Dup led to ectopic interactions between the Fgf8 AER enhancers and two other genes in the locus, Lbx1 and Btrc, resulting in their misexpression in the AER in a typical Fgf8 pattern. In contrast, Fgf8 expression remained unchanged. This was due to the fact that Fgf8 was not included in the duplication and a fully intact Fgf8 regulatory landscape remained, ensuring normal expression. The duplication resulted in the formation of a neo-TAD which consisted of the truncated Fgf8 TAD and a small region upstream of the centromeric $L b \times 1$ TAD boundary. As a consequence, this neo-TAD contained only Fbwx4 with the AER enhancers and would thus not be expected to give rise to any 
phenotypic consequences. However, the Fgf8 enhancers within the neo-TAD were now devoid of their target gene Fgf8. This was likely to result in increased interaction with other possible partners such as $L b x 1$ and $B t r c$, potentiated by a pre-existing low frequency interaction between the domains, by a weaker TAD boundary upon rearrangement and also by the shorter distance particularly with $L b x 1$.

In a screen for structural variations in a cohort of patients with limb malformations, we also identified an inversion at the SHFM3 locus. Our data showed that the inversion (Inv1) resulted in a shift of regulatory elements from the Fgf8 TAD into the $L b x 1$ TAD thereby giving rise to the equivalent regulatory effect as the SHFM duplications. Interestingly, as for the duplication, the inversion involved the four essential Fgf8 AER enhancers (CE58, CE59, CE61, CE66) that are located in the introns of the Fbxw4 gene (Fig. 1a and 1b). Previous experiments demonstrated that a loss of Fgf8 expression upon deletion of the four distal enhancers resulted in similar defects as conditional ablation of $F g f 8$ in the $\operatorname{limb}^{24,25}$. In Inv1 we also observed a loss of $F g f 8$ expression and the corresponding Fgf8 loss of function phenotype. This time, however, the enhancer region was intact but relocated from the Fgf8 TAD into the $L b x 1$ TAD. Thus, a repositioning of the boundary was sufficient to disconnect Fgf8 from its enhancers resulting in a loss of Fgf8 expression in the limb. The effect of Inv1 was thus similar to the reported inversion at the Sox9/Kcnj2 locus which resulted in a loss of Sox9 expression due to a swap of Sox9 regulatory elements from the Sox9 TAD to the Kcnj2 $\mathrm{TAD}^{33}$. In both SHFM-associated rearrangements, only Lbx1 and Btrc were misexpressed in the AER. Poll, Dpcd and Fbxw4 showed very low levels of expression in these cells. This indicated that these genes were not involved in SHFM3. 
Our interpretation that $L b x 1$ and Btrc were ectopically activated by Fgf8 AER enhancers was further supported by our AER enhancers insertion experiment within the Lbx1 TAD (Fgf8 AER enh $K I)$. As in the duplication and in the inversion, the Fgf8 AER enhancers were able to activate both Btrc and $L b x 1$, with the latter being more affected. The compatibility between $L b x 1$ and the Fgf8 AER enhancers could be also explained with the fact that both $L b x 1$ and Fgf8 are developmental genes and active in the same tissue. Given a certain degree of leakiness of the Lbx1/Fgf8 boundary, the contacts between the Fgf8 AER enhancers and $L b x 1$ and Btrc might also be expected to lead to misexpression of Btrc and $L b \times 1$ in wild-type cells. However, no crossactivation takes place. One possible explanation is that a certain threshold needs to be reached to result in gene activation and this is not achieved in the wild-type situation. In $\ln v 1$ the level of interactions was favoured by the repositioning of the Fgf8 AER enhancers within the Lbx1 TAD, while in Dup the Fgf8 AER enhancers in the neo-TAD lose their natural target promoter (Fgf8), which is not part of the neo-TAD as not included in the SV. This is likely to cause a further increase in the frequency of contacts across the leaky Lbx1/Fgf8 boundary on the centromeric side as well as the newly positioned boundary telomeric of the neo-TAD, with subsequent misexpression of Lbx1 and Btrc.

In Inv1 we were able to partially recapitulate the SHFM phenotype. However, the precise pathomechanism through which Btrc and $L b x 1$ cause the phenotype is still unclear. The Dactylaplasia mouse mutant displays a similar phenotype to the one observed in some SHFM3 patients and the two corresponding alleles (Dac1j and Dac2j) map in the region with the duplications and inversion in SHFM3 ${ }^{34}$. Based on these similarities, the mutant has long been thought as a model for human SHFM3, but Dac1j and Dac2 $j$ were shown to be associated with insertions of a MusD 
retrotransposon upstream of the Fbxw4 gene and within intron 5 of Fbxw4, respectively ${ }^{34}$. The absence of any structural variations in these mutants and the differences between the nature of the human and mouse genomic abnormalities argue against a common pathogenesis. However, both the mouse and the human mutations involve the regulatory elements located within and around the Fbxw4 gene possibly providing a common pathogenetic mechanism.

Similar to the reported misexpression of Pax3 in an Epha4-like pattern in the limb in brachydactyly ${ }^{3}$, misexpression of the muscle-specific transcription factor $L b x 1$ in the AER is likely to have effects on gene expression and thus the functionality of the AER. This interpretation is supported by our finding that $L b \times 1$ misexpression results in the activation of muscle genes/pathways in the AER cells. At the same time, we cannot exclude a potential contribution of a loss of Fgf8 expression to the Inv1 mutant phenotype. The AER is the major signalling center for proximodistal growth and distal limb development. It is induced and maintained through the reciprocal interactions between the ectoderm and the underlying mesenchyme involving Wnt$B m p-F g f$ signaling pathways ${ }^{35}$. Disruption or malfunction of the AER results in diminished growth and thus hypoplasia/aplasia of digits, thus correlating with the SHFM phenotype ${ }^{36}$. Our results support the concept that SHFM is caused by AER defects, and in SHFM3 by gene misexpression. Mice are commonly used as a model organism to study human disease because of the similarities in genetics, physiology and organ development. However, inter-species differences exist and account for genotype-to-phenotype divergences between mouse and human ${ }^{37}$. When comparing CTCF binding sites in mouse and human at the FGF8 locus, we noticed that the centromeric $L b \times 1$ TAD boundary is missing one binding site in reverse orientation in human in comparison to mouse (black arrow in Fig. 3a). This additional binding site in mouse could thus interfere with the levels 
of ectopic interactions between the Fgf8 AER enhancers and Lbx1 and Btrc in the Dup mutant, consequently reducing the level of misexpression in the AER. Indeed, gene dosage effects related to the rearrangements may explain the absence of a striking phenotype in the Dup in comparison to Inv1 which is associated with highest levels of $L b \times 1$ and Btrc misexpression, causing a mouse phenotype similar to the human phenotype.

Overall, this study offers new insights into: i) the molecular phenotype of SHFM3-associated duplications and inversion, ii) gene regulation at the $L b x 1 / F g f 8$ locus in the context of $3 D$ genome architecture and, iii) the consequences deriving from perturbations of the local chromatin structure. Notably, we provide a new and complex scenario by which SVs can cause disease. Indeed, we report in this study an example of SVs causing disease through a combined position effect mechanism resulting in ectopic gene misexpression involving multiple genes and a dosedependent effect. To the best of our knowledge, the SVs-associated pathogenic mechanism reported in this study has not been shown previously.

In the era of whole genome sequencing (WGS) and the increased number of detected SVs, the medical interpretation of SVs and the prediction of their phenotypic consequences remain unsatisfactory. Thus, this study provides a conceptual framework when interpreting the pathogenic potential of these variant types. 


\section{MATERIAL AND METHODS}

\section{Mouse embryonic stem cell targeting}

Culture and genome editing of mouse embryonic stem cells (mESCs) was performed as described previously ${ }^{21,33}$. The size and position of the human structural variations (hg19) were converted to the mouse genome (mm9) using the UCSC liftOver tool. All clones were genotyped by PCR and qPCR analyses. Positive clones were expanded, their genotyping further confirmed by PCR, qPCR and Sanger sequencing of PCR products, and used for further experiments only if the successful modification could be verified. A list of single guide RNAs (sgRNAs) used for CRISPR/Cas9 genome editing and of all the primers used for genotyping is given in Supplementary Table 1. For the Fgf8-AER-enh-KI mutant, a 6993bp DNA fragment containing the 4 Fgf8-AER enhancers with a deletion of the CTCF site in enhancer CE58 was synthetised and subsequently cloned into a pUC vector by Genewizz. Homology arms targeting the $L b x 1$ locus were then cloned using Gibson assembly and the resulting vector was transfected in mESCs together with the pX459-sgRNA for CRISPR/Cas9 homology-directed-repair (HDR).

\section{Generation of mice}

Mice were generated from genome-edited mESCs by diploid or tetraploid aggregation ${ }^{42}$ and genotyped by PCR and qPCR analysis. All animal procedures were conducted as approved by the local authorities (LAGeSo Berlin) under license numbers G0243/18 and G0176/19.

\section{Whole mount in situ hybridization (WISH)}


RNA expression in E11.5 mouse embryos from wild-type and mutants was assessed by WISH using digoxigenin (DIG)-labelled antisense riboprobes for Lbx1, Btrc, Poll, Dpcd, Fbxw4 and Fgf8 transcribed from linearized gene-specific probes (PCR DIG Probe Synthesis Kit; Roche). Primers for probe generation are listed in Supplementary Table 1. Embryos were collected and fixed overnight in 4\% PFA in PBS, then washed twice for 30 min in PBS with $0.1 \%$ Tween (PBST), dehydrated for 30 min each in 25\%, 50\% and $75 \%$ methanol in PBST and stored at $-20{ }^{\circ} \mathrm{C}$ in $100 \%$ methanol. For WISH, genotyped embryos were rehydrated on ice in reverse methanol/PBST steps, washed twice in PBST, bleached in $6 \% \mathrm{H}_{2} \mathrm{O}_{2}$ in PBST for $1 \mathrm{~h}$ and washed in PBST. Embryos were washed in PBST and refixed for 20 min with 4\% PFA in PBS, 0.2\% glutaraldehyde and 0.1\% Tween. After further washing steps with PBST, embryos were incubated at $68^{\circ} \mathrm{C}$ in L1 buffer $(50 \%$ deionized formamide, $5 \times$ SSC, $1 \%$ SDS, $0.1 \%$ Tween 20 in diethyl pyrocarbonate (DEPC), pH 4.5) for $10 \mathrm{~min}$. For prehybridization, embryos were incubated for $2 \mathrm{~h}$ at $68^{\circ} \mathrm{C}$ in hybridization buffer 1 (L1 with $0.1 \%$ tRNA and $0.05 \%$ heparin), while for subsequent probe hybridization, embryos were incubated overnight at $68^{\circ} \mathrm{C}$ in hybridization buffer 2 (hybridization buffer 1 with $0.1 \%$ tRNA, $0.05 \%$ heparin and 1:500 DIG probe). The next day, unbound probes were washed away through repeated washing steps: $3 \times 30 \mathrm{~min}$ at $68^{\circ} \mathrm{C}$ with $\mathrm{L} 1, \mathrm{~L} 2$ (50\% deionized formamide, $2 \times$ SSC, pH 4.5, 0.1\% Tween 20 in DEPC, pH 4.5) and L3 (2× SSC, pH 4.5, 0.1\% Tween 20 in DEPC, pH 4.5). For signal detection, embryos were treated for $1 \mathrm{~h}$ with RNase solution $(0.1 \mathrm{M} \mathrm{NaCl}, 0.01 \mathrm{M}$ Tris, $\mathrm{pH} 7.5,0.2 \%$ Tween 20, $100 \mu \mathrm{g} \mathrm{m}^{-1}$ RNase A in $\mathrm{H}_{2} \mathrm{O}$ ), followed by washing in TBST 1 (140 mM $\mathrm{NaCl}, 2.7 \mathrm{mM} \mathrm{KCl}, 25 \mathrm{mM}$ Tris $-\mathrm{HCl}, 1 \%$ Tween 20, $\mathrm{pH} 7.5$ ) and blocking for $2 \mathrm{~h}$ at room temperature in blocking solution (TBST 1 with $2 \%$ calf serum and $0.2 \%$ bovine serum albumin). Overnight incubation with Anti-Dig antibody conjugated to alkaline phosphatase $(1: 5,000)$ at $4{ }^{\circ} \mathrm{C}$ 
(no. 11093274910; Roche) was followed by $8 \times 30$ min washing steps at room temperature with TBST 2 (TBST with $0.1 \%$ Tween 20 and $0.05 \%$ levamisole-tetramisole) and left overnight at $4{ }^{\circ} \mathrm{C}$. Embryos were finally stained after equilibration in AP buffer $(0.02 \mathrm{M} \mathrm{NaCl}, 0.05 \mathrm{M} \mathrm{MgCl}, 0.1 \%$ Tween 20, $0.1 \mathrm{M}$ Tris $-\mathrm{HCl}$ and $0.05 \%$ levamisole-tetramisole in $\mathrm{H}_{2} \mathrm{O}$ ) $3 \times 20 \mathrm{~min}$, followed by staining with BM Purple AP Substrate (Roche). The stained embryos were imaged using a Zeiss SteREO Discovery V12 microscope and Leica DFC420 digital camera.

\section{Skeletal preparation}

E18.5 fetuses were kept in $\mathrm{H} 2 \mathrm{O}$ for $1-2 \mathrm{~h}$ at room temperature and heat shocked at $65^{\circ} \mathrm{C}$ for 1 min. The skin was gently removed, together with the abdominal and thoracic viscera using forceps. The fetuses were then fixed in $100 \%$ ethanol overnight. Afterwards, alcian blue staining solution (150 mg I-1 alcian blue $8 \mathrm{GX}$ in $80 \%$ ethanol and $20 \%$ acetic acid) was used to stain the cartilage overnight. After $24 \mathrm{~h}$ fetuses were rinsed and postfixed in $100 \%$ ethanol overnight, followed by $24 \mathrm{~h}$ incubation in $0.2 \% \mathrm{KOH}$ in $\mathrm{H} 2 \mathrm{O}$ for initial clearing. The next day fetuses were incubated in alizarin red ( $50 \mathrm{mg} \mathrm{l}-1$ alizarin red $\mathrm{S}$ in $0.2 \%$ potassium hydroxide) to stain the bones overnight. Following this, rinsing and clearing was done for several days using $0.2 \% \mathrm{KOH}$. The stained embryos were dissected in $25 \%$ glycerol, imaged using a Zeiss SteREO Discovery V12 microscope and Leica DFC420 digital camera, and subsequently stored in $80 \%$ glycerol.

\section{Capture Hi-C}

E11.5 mouse limb buds were prepared in 1x PBS and dissociated by trypsin treatment for $10 \mathrm{~min}$ at $37^{\circ} \mathrm{C}$, pipetting every 2 minutes to obtain a single-cell suspension. Trypsin was stopped by 
adding $5 x$ volume of $10 \%$ FCS/PBS. The solution was then filtered through $40 \mu \mathrm{m}$ cell strainer (Falcon) to remove cell debris, cells were centrifuged at $1100 \mathrm{rpm}$ for $5 \mathrm{~min}$ and the pellet was then resuspended in $5 \mathrm{ml} \mathrm{10 \%} \mathrm{FCS/} \mathrm{PBS.} 5 \mathrm{ml}$ of freshly prepared $4 \%$ formaldehyde in $10 \%$ FCS/PBS (final concentration 2\%) were added to perform crosslinking and samples were incubated in rotation for $10 \mathrm{~min}$ at room temperature. The crosslinking reaction was stopped by adding $1 \mathrm{ml} 1.425 \mathrm{M}$ glycine on ice, followed by centrifugation at $1500 \mathrm{rpm}$ and $4^{\circ} \mathrm{C}$ for $8 \mathrm{~min}$, pellet resuspension in $5 \mathrm{ml}$ freshly prepared, cold lysis buffer (final concentration of $10 \mathrm{mM}$ Tris pH 7.5, $10 \mathrm{mM} \mathrm{NaCl}, 5 \mathrm{mM} \mathrm{MgCl} 2,0.1 \mathrm{M}$ EDTA, and $1 \times$ cOmplete $^{\text {TM }}$ protease inhibitors (SigmaAldrich) and incubation for at least $10 \mathrm{~min}$ on ice. Nuclei were pelleted by centrifugation at 2000 $\mathrm{rpm}$ at $4^{\circ} \mathrm{C}$ for $5 \mathrm{~min}$, washed with $1 \times$ PBS, aliquoted in $1.5 \mathrm{ml}$ tubes with $2.5-5 \times 10^{6}$ nuclei each, followed by supernatant removal, snap-freezing and storage at $-80^{\circ} \mathrm{C}$.

For the 3C library preparation, the nuclei pellet was resuspended in $360 \mu$ l of water, mixed with $60 \mu$ of 10x Dpnll buffer (Thermo Fisher Scientific) and incubated for $5 \mathrm{~min}$ at $37^{\circ} \mathrm{C}$ in a thermomixer at $900 \mathrm{rpm} .15 \mu \mathrm{l}$ of $10 \%$ SDS was then added and the samples were incubated for $1 \mathrm{~h}$ at $37^{\circ} \mathrm{C}$ and $900 \mathrm{rpm}$, with occasional pipetting to help dissolving the nuclei aggregates. Next, $150 \mu$ l of $10 \%$ Triton X-100 were added, followed by $1 \mathrm{~h}$ of incubation at $37^{\circ} \mathrm{C}$ and $900 \mathrm{rpm}$. After adding $600 \mu \mathrm{l}$ of $1 \times$ Dpnll buffer, a $10 \mu \mathrm{l}$ aliquot was taken as undigested control and stored at $4^{\circ} \mathrm{C}$ and 400 units of restriction enzyme Dpnll were added to the samples. After $4 \mathrm{~h}$ of digestion additional 200 units of Dpnll enzyme were added and the samples were incubated overnight at $37^{\circ} \mathrm{C}$ with shaking at $900 \mathrm{rpm}$. After overnight incubation, a $10 \mu \mathrm{l}$ aliquot was taken as digested control and stored at $4^{\circ} \mathrm{C}$, while the samples were supplemented with further 200 units of DpnII enzyme for additional four hours. Meanwhile, the undigested an digested controls were tested. 
For this, each $10 \mu \mathrm{l}$ aliquot was mixed with $85 \mu \mathrm{l} 10 \mathrm{mM}$ Tris $\mathrm{pH} 7.5$ and $2 \mu \mathrm{l}$ Rase $\mathrm{A}(10 \mathrm{mg} / \mathrm{ml}$ ) and incubated for $1 \mathrm{~h}$ at $37^{\circ} \mathrm{C}$. Chromatin was decrosslinked by adding $5 \mu$ l proteinase $\mathrm{K}(10 \mathrm{mg} /$ $\mathrm{ml}$ ) and incubation at $65^{\circ} \mathrm{C}$ for $4 \mathrm{~h}$. The DNA was then extracted by adding $100 \mu \mathrm{l}$ phenolchloroform. Samples were mixed by inverting the tubes and centrifuged for $10 \mathrm{~min}$ at $13200 \mathrm{rpm}$ at room temperature. The upper water phase was transferred into new $1.5 \mathrm{ml}$ tubes and analysed on a $1 \%$ agarose gel. After correct validation, restriction enzyme in the original samples still under digestion was heat-inactivated at $65^{\circ} \mathrm{C}$ for $20 \mathrm{~min}$. The samples were transferred to $50 \mathrm{ml}$ Falcon tubes and $700 \mu \mathrm{l} 10 \mathrm{x}$ ligation buffer (Thermo Fisher Scientific) were added. The volume was filled up to $7 \mathrm{ml}$ with water and 50 units of T4 DNA ligase (Thermo Fisher Scientific) were added. The ligation mix was incubated overnight at $4^{\circ} \mathrm{C}$ in rotation. The next day, a $100 \mu$ l aliquot of religated DNA was collected and analysed on an agarose gel as described above. Upon successful ligation, samples were de-crosslinked by adding $30 \mu \mathrm{l}$ of proteinase $\mathrm{K}(10 \mathrm{mg} / \mathrm{ml})$ and incubating overnight at $65^{\circ} \mathrm{C}$. Then, $30 \mu \mathrm{l}$ RNase $\mathrm{A}(10 \mathrm{mg} / \mathrm{ml})$ were added and the samples were incubated for $45 \mathrm{~min}$ at $37^{\circ} \mathrm{C}$. The DNA was then extracted by adding $7 \mathrm{ml}$ phenol-chloroform. The solution was mixed by inverting the tube and the water phase was separated by centrifugation at $3750 \mathrm{rpm}$ for $15 \mathrm{~min}$ at room temperature. DNA was precipitated by adding the following reagents to the water phase: $7 \mathrm{ml}$ water, $1.5 \mathrm{ml} 2 \mathrm{M} \mathrm{NaAc} \mathrm{pH}$ 5.6, $140 \mu \mathrm{g}$ glycogen, 35 $\mathrm{ml} 100 \%$ ethanol. The solution was mixed and placed at $-80^{\circ} \mathrm{C}$ overnight or until the samples were completely frozen. The sample was then thawed and centrifuged for $20 \mathrm{~min}$ at $8350 \mathrm{~g}$ and $4^{\circ} \mathrm{C}$. DNA pellet was washed with $30 \mathrm{ml}$ cold $70 \%$ ethanol and centrifuged $15 \mathrm{~min}$ at $3300 \mathrm{~g}$ at $4^{\circ} \mathrm{C}$. Dried pellet was dissolved in $150 \mu \mathrm{l} 10 \mathrm{mM}$ Tris $\mathrm{pH} 7.5$ at $37^{\circ} \mathrm{C}$. The final $3 \mathrm{C}$ library was checked on $1 \%$ agarose gel and subsequently used for capture $\mathrm{Hi}-\mathrm{C}$ preparation. $3 \mathrm{C}$ libraries were 
sheared using a Covaris sonicator (duty cycle: 10\%; intensity: 5; cycles per burst: 200; time: 6 cycles of $60 \mathrm{~s}$ each; set mode: frequency sweeping; temperature: $4-7^{\circ} \mathrm{C}$ ). Adaptors were added to the sheared DNA and amplified according to Agilent instructions for Illumina sequencing. The library was hybridized to the custom-designed SureSelect beads and indexed for sequencing (50 bp paired-end) following Agilent instructions. SureSelect enrichment probes were designed over the genomic interval chr19:44,440,000-46,400,000 (mm9) using the online tool of Agilent: SureDesign. Probes were covering the entire genomic region and were not designed specifically in proximity of Dpnll sites. Samples were sequenced with Illumina Hi-Seq technology according to standard protocols.

\section{Capture Hi-C analysis}

In a pre-processing step, the reads in fastq files were trimmed to $50 \mathrm{bp}$, if necessary, to obtain the same initial read length for all samples. Afterwards, mapping, filtering and deduplication of paired-end sequencing data was performed using the HiCUP pipeline v0.8.1(PMID: 26835000) (no size selection, Nofill: 1, Format: Sanger). The pipeline was set up with Bowtie2 v2.4.2 (PMID: 22388286) for mapping short reads to reference genome mm9. For merging biological replicates, the final bam files produced by the HiCUP pipeline were joined. Juicer tools v1.19.02 (PMID: 27467249) was used to generate binned and KR normalized ${ }^{43}$ contact maps from valid and deduplicated read pairs. For the generation of $\mathrm{cHi}-\mathrm{C}$ maps, only read-pairs referring to the region of interest (chr19:44,440,001-46,400,000) and with $M A P Q \geq 30$ were considered. KR normalized maps were exported for $10 \mathrm{~kb}$ bin size. For both inversions, a custom reference genome was derived from mm9, which considered the corresponding inversion. For the samples with a 
duplication it is noted, that the matrix balancing of the KR normalization affects the signal in the duplicated region, because it scales down the signal intensities in this region to fit to the other regions of the cHi-C map. Subtraction maps were computed from KR-normalized cHi-C maps, which were normalized in a pairwise manner before subtraction as follows. To account for differences between two maps in their distance-dependent signal decay, the maps were scaled jointly across their sub-diagonals. Therefore, the values of each sub-diagonal of one map were divided by the sum of this sub-diagonal and multiplied by the average of these sums from both maps. Afterwards, the maps were scaled by $10^{6} /$ total sum. For the computation of scaling factors, the duplicated as well as the inverted regions were excluded in both maps. $\mathrm{cHi}-\mathrm{C}$ maps were visualized as heatmaps with linear scale, with very high values being truncated to improve visualization.

\section{Virtual 4C profiles}

Virtual 4C-like interaction profiles were generated for individual viewpoints from the same bam files also used for the $\mathrm{cHi}-\mathrm{C}$ maps. Paired-end reads with $\mathrm{MAPQ} \geq 30$ were considered in a profile, when one read mapped to the defined viewpoint region and the other one outside of it. Contacts of a viewpoint region were counted per restriction fragment. The count profile was binned to a $1 \mathrm{~kb}$ grid. In case a fragment overlapped more than one bin, the counts were distributed proportionally. Afterwards, the binned profile was smoothed by averaging within a sliding window of $5 \mathrm{~kb}$ size and scaled by $10^{3} /$ sum of counts within the enriched region. The viewpoint region itself and a window $\pm 5 \mathrm{~kb}$ were excluded from the computation of the scaling factor. 
Interaction profiles were generated with custom Java code using v2.12.0 (https://samtools.github.io/htsjdk/).

\section{RNA-seq}

E11.5 limb buds were microdissected from wild-type and mutant embryos $(n=3)$ and immediately frozen in liquid nitrogen. Total RNA was isolated using the RNeasy Mini Kit (QIAGEN). Samples were poly-A enriched and sequenced (paired-end $50 \mathrm{bp}$ ) using Illumina technology following standard protocols.

\section{RNA-seq data processing}

Reads were mapped to the mouse reference genome $(\mathrm{mm} 9)$ using the STAR mapper ${ }^{44}$ (splice junctions based on RefSeq; options: -alignIntronMin 20-alignIntronMax 500000outFilterMultimapNmax 5-outFilterMismatchNmax $\quad$ 10-outFilterMismatchNoverLmax 0.1 ). Reads per gene were counted as described previously ${ }^{3}$, and used for differential expression analysis with the DEseq2 package ${ }^{45}$.

\section{scRNA-seq}

scRNA-seq experiments were performed in single replicates that were jointly processed to avoid batch effects. E11.5 hindlimb buds of wild-type and Dup embryos were microdissected in 1xPBS at room temperature. A single-cell suspension was obtained by incubating the tissue for $10 \mathrm{~min}$ at $37^{\circ} \mathrm{C}$ in $200 \mu \mathrm{l} \mathrm{Gibco} \mathrm{trypsin-EDTA} 0.05 \%$ (Thermo Fisher Scientific) supplemented with $20 \mu \mathrm{l}$ 5\% BSA (Sigma-Aldrich). Trypsinization was then stopped by adding $400 \mu$ of $5 \%$ BSA. Cells were 
then resuspended by pipetting, filtered using a $0.40 \mu \mathrm{m}$ cell strainer (Falcon), washed once with $0.04 \% \mathrm{BSA}$, centrifuged for $5 \mathrm{~min}$ at $1200 \mathrm{rpm}$, then resuspended in $0.04 \% \mathrm{BSA}$. The cell concentration was determined using an automated cell counter (Bio-Rad) and cells were subjected to scRNA-seq (10x Genomics, Chromium Single Cell 3' v2; one reaction per time point and per strain has been used) aiming for a target cell recovery of up to 10,000 sequenced cells per sequencing library (time point and strain). Single-cell libraries were generated according to the 10x Genomics instructions with the following conditions: 8 PCR cycles were run during cDNA amplification and 12 PCR cycles were run during library generation and sample indexing to increase library complexity. Libraries were sequenced with a minimum of 230 million paired end reads.

\section{scRNA-seq data processing}

The Cell Ranger pipeline v.3 (10x Genomics Inc.) was used for each scRNA-seq sample in order to de-multiplex the raw base call files, to generate the fastq files, and to perform the alignment against a custom mouse reference genome $\mathrm{mm} 9$ to create the unique molecular identifier (UMI) count matrix. Only cells with more than 2000 detected genes and less than $10 \%$ of mitochondrial UMI counts were considered for further analysis. In addition, Scrublet ${ }^{46}$ was used to identify potential doublets in our dataset. Cells with a Scrublet score higher than 0.2 were filtered out. Each sample was normalized independently using the SCT method ${ }^{47}$ implemented in Seurat $3^{48} \mathrm{R}$ package and then, these were integrated using the Seurat3 IntegrateData CCA-based (canonical correlation analysis) approach considering the top 2,000 most variable genes. The first 20 principal components of this joint dataset were calculated and used for UMAP projection and 
reconstruction of the cell-cell similarity graph. To delimit the major hindlimb bud cell types, we used the Louvain algorithm implemented in the Seurat3 function FindClusters and the expression of well-known marker genes for the limb-comprising cell types. For the AER cluster, we used the marker gene Fgf8. Differential gene expression between the WT and the Dup cells was estimated by modelling the gene expression as a function of the genotype across cells. We fitted a quasiPoisson distribution to calculate the effect of the Dup genotype on the gene expression distribution of each gene using the monocle3 strategy ${ }^{49}$. We tested that such effect was not equal to 0 . We use this condition association effect to rank the genes and identified the pathways associated with the top 200 genes using the Enrichr tool from the Maayan lab ${ }^{50}$. Finally, we confirmed that the top perturbed genes associated to the muscle pathways are more frequently expressed in the Dup AER cells.

\section{C-seq}

For 4C-seq libraries, $5 \times 10^{6}-10^{7}$ cells were used. The fixation and lysis were performed as described in the Capture Hi-C section. After the first digestion with DpnII (NEB), sticky ends were religated in a $50 \mathrm{ml}$ falcon tube (700 $\mu \mathrm{l} 10$ ligation buffer (Fermentas), $7 \mathrm{ml} \mathrm{H2O,} 50 \mathrm{U}$ T4 DNA ligase (Thermo); overnight at $16{ }^{\circ} \mathrm{C}$ ) and DNA de-cross linked and cleaned as described in the Capture Hi-C section. Next, a second digestion (150 $\mu$ l sample, $50 \mu$ l0x Csp6I buffer (Thermo), $60 \mathrm{U}$ Csp6I (Thermo) $295 \mu \mathrm{l} \mathrm{H2O}$; overnight at $37^{\circ} \mathrm{C}$ ) and another re-ligation were performed. For all viewpoints, DNA was purified using a PCR clean up Kit (Qiagen) and 1.6 $\mu$ g DNA was amplified by PCR (LBX1 Viewpoint: read-primer 5'-TCTATATGCTACCATGATC-3', secondary-primer 5'GATGAACTGGAATACCCA-3'; FGF8 Viewpoint: read-primer 5'-AGGGTGCGTTCCAAGATC-3', 
secondary-primer 5'-GGTGGCCTGGATGGAAGT-3'; BTRC Viewpoint: read-primer 5'CAACGCAGCGCCCGGATC-3', secondary-primer 5'-CTGGGAATGAGGACCTAGGGC-3'). For the library reaction, primers were modified with TruSeq adapters (Illumina): Adapter1 5'CTACACGACGCTCTTCCGATCT-3' and Adapter2 5'-CAGAC GTGTGCTCTTCCGATCT-3'. Between 50 and 200 ng were used as input of a single 4C PCR reaction depending on the complexity. The reaction was performed in a $50 \mu \mathrm{l}$ volume using the Expand Long Template System (Roche) and 29 reaction cycles. After the PCR all reactions were combined and the DNA purified with a PCR clean up Kit (Qiagen). All samples were then sequenced with the HiSeq 4000 (Illumina) technology according to the standard protocols and with around 20 million single- end reads per sample. Sequencing reads were mapped, normalized and smoothed with pipe $4 \mathrm{C}^{51}$ using the reference genome GRCh37 and default settings. All viewpoints were performed in replicates and as quality measure $>70 \%$ of reads were mapped within a size range of $1 \mathrm{Mb}$ and $>80 \%$ within $100 \mathrm{~kb}$ around the viewpoint.

\section{Human material}

Skin biopsies were collected from SHFM3 patients and controls by standard procedures. Fibroblasts were cultured in DMEM (Lonza) supplemented with 10\% fetal calf serum (Gibco), 1\% L-glutamine (Lonza) and 1\% penicillin/streptomycin (Lonza). Informed consent was obtained from all individuals that participated in this study. This study was approved by the Charité Universitätsmedizin Berlin ethics committee. 


\section{ACKNOWLEDGEMENTS}

This study was supported by a grant from the Deutsche Forschungsgemeinschaft (MU 880/22-1) to S.M.

We thank the transgenic unit, sequencing core and animal facility of Max Planck Institute for Molecular Genetics for technical assistance, Ute Fischer for technical support and Norbert Brieske for help with whole mount in situ hybridizations and image processing. We would like to thank all members of the Mundlos laboratory and Tugce Aktas for continuous support and discussion. We would like to particularly thank Dario Lupiáñez, Chiara Anania, Lila Allou and Jane Skok for critical reading of the manuscript.

\section{AUTHOR CONTRIBUTIONS}

G.C., S.M. and M.S. conceived the project. G.C. performed most experiments with major support from J.G and further technical support of M.F., R.F. and M.F. L.W. performed morula aggregation. R.S. performed cHi-C computational analysis and generated virtual 4C maps. S.A. performed and analysed 4C-seq. C.A.P.-M. performed Single Cell RNA-seq computational analysis. B.T. oversaw high-throughput sequencing. M.S., E.P., R.F., F.N., M.G., and O.Z. examined the patients, interpreted the clinical data and provided the clinical information together with biological samples. G.C. and S.M. wrote the manuscript with input from all the authors.

\section{COMPETING INTERESTS}

The authors declare no competing interests. 


\section{FIGURES}

a

2010

Wild-type

$500 \mathrm{~kb}$
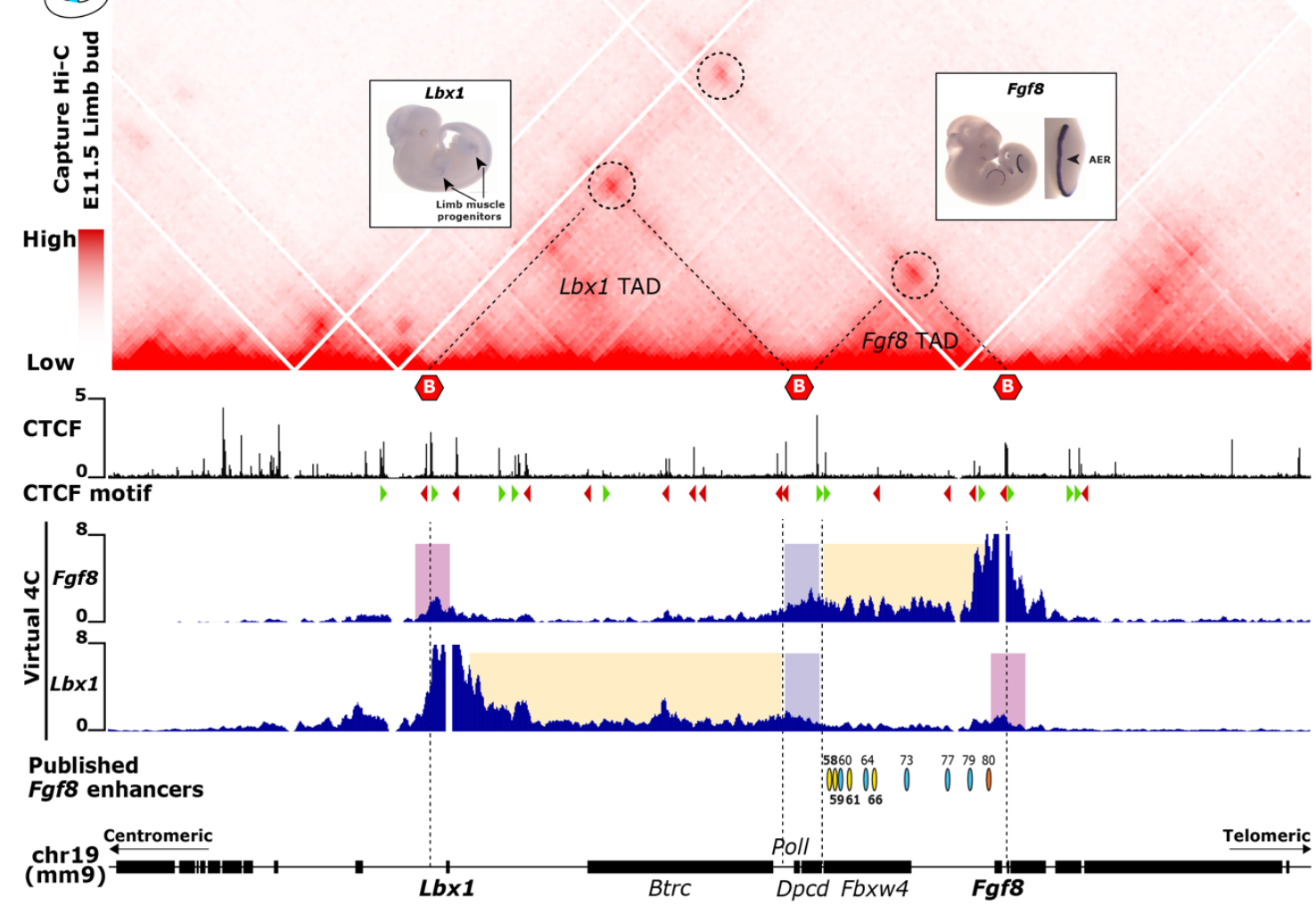

b Human

SHFM3 SVs

1111 | 11111 Duplications

Inversion

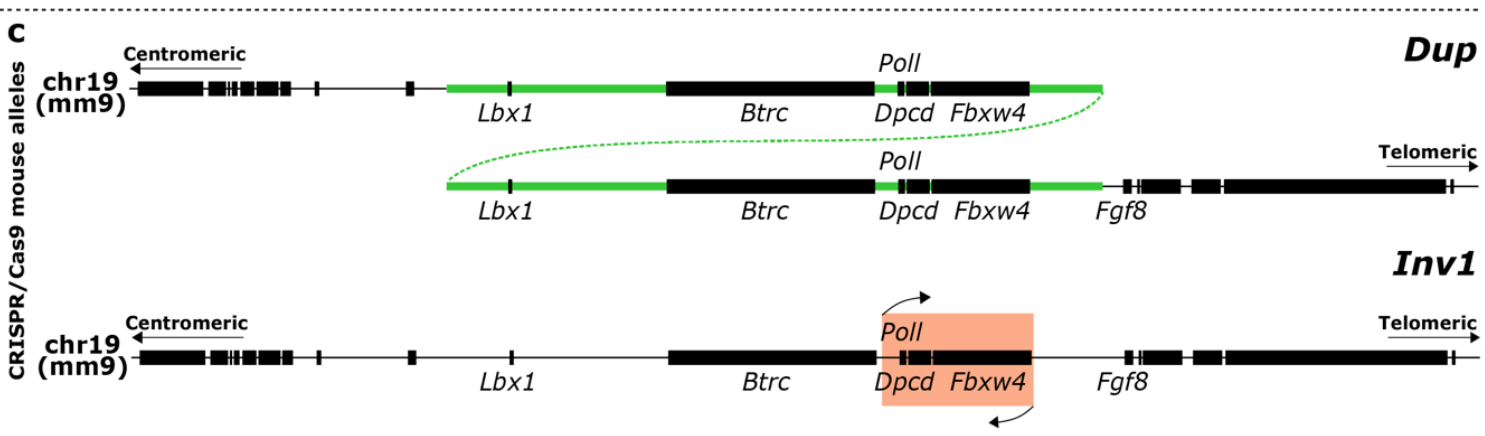

Fig. 1 | 3D chromatin architecture at the Fgf8 locus and localisation of SHFM-associated SVs. a, cHi-C of extended Lbx1/Fgf8 locus generated from wild-type E11.5 mouse limb buds. The region consists of two TADs (indicated by dashed lines) separated by boundaries (indicated by red hexagons) in correlation with CTCF binding sites, as indicated in ChIP-seq from E11.5 mouse limb buds ${ }^{38}$ below. Loops (dashed circles) indicate interaction between CTCF sites. Interaction plots using virtual $4 C$ from Fgf8 and $L b x 1$ viewpoints are shown below. Regions of interactions restricted only to Lbx1 and Fgf8 within their own TADs are highlighted in yellow, whereas contacts with the boundary region between $L b \times 1$ and Fgf8 TADs and those over such boundary are in lilac and pink, respectively. Published $F g f 8$ enhancers ${ }^{25}$ are indicated by ovals. 
Yellow ovals highlight enhancers driving Fgf8 expression in the AER and localized within the introns of Fbxw4, while in orange is the only AER enhancer in close proximity to Fgf8. b, Schematic of human SHFM3 related structural variations (SVS). Red and blue lines in the duplications bar represent the different centromeric and telomeric breakpoints, respectively. Breakpoints of the inversion are shown below. c, Schematic of the SHFM-associated SVs engineered in mice using CRISPR/Cas9 genome editing tool, particularly one selected tandem duplication (Dup) and the inversion (Inv1). 

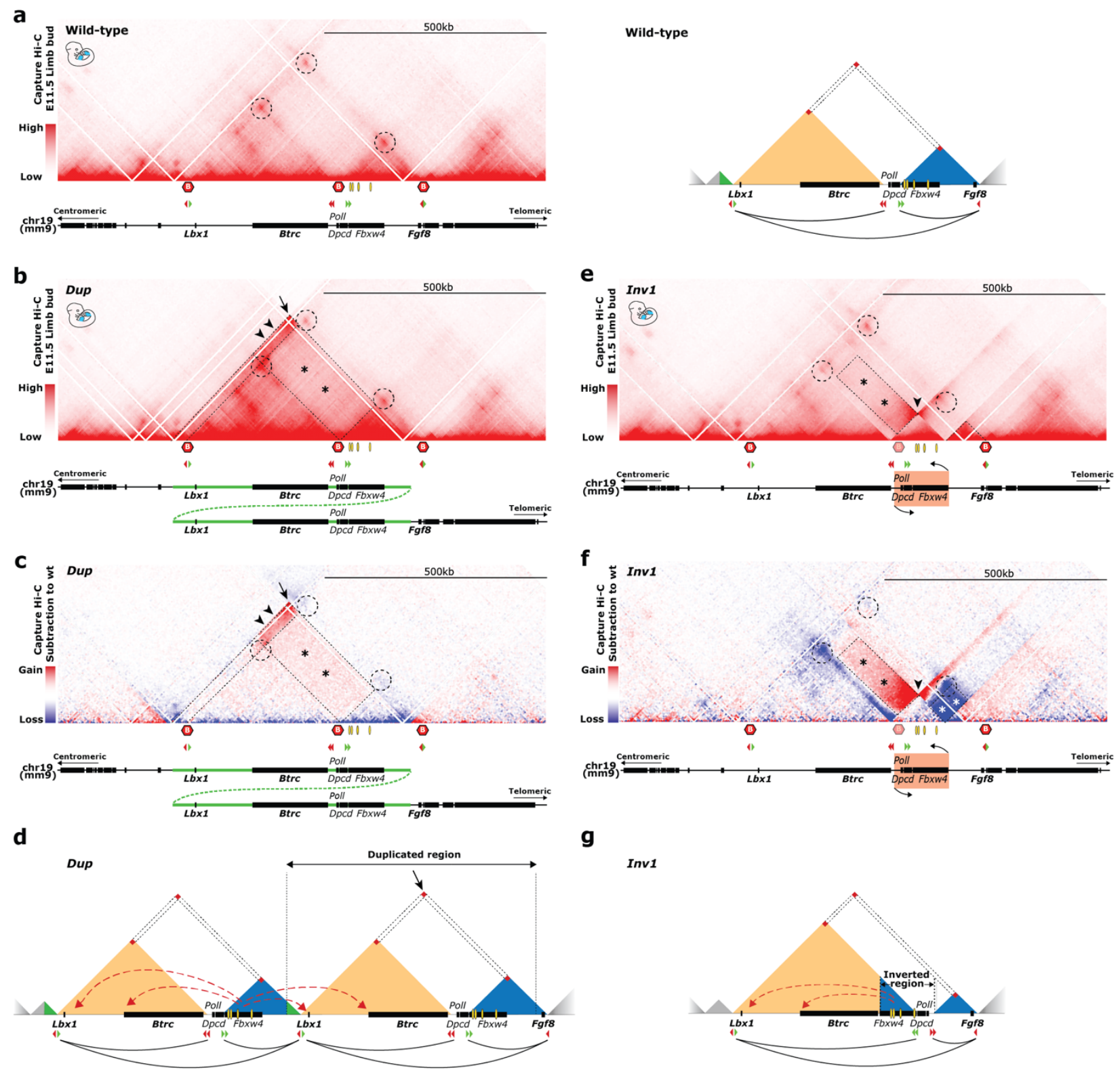

Fig. 2 | Ectopic interactions upon structural rearrangements at the Lbx1/Fgf8 locus. a, Wild-type cHi-C from E11.5 mouse limb buds (left panel). Boundaries are indicated by red hexagons and interactions between boundaries are highlighted by dashed circles. Orientation of CTCF binding sites and schematic of the locus are shown below. Yellow ovals highlight the Fgf8 AER enhancers. Schematic of wild-type configuration is shown on the right (right panel). Lbx1 TAD in yellow, Fgf8 TAD in blue. Interactions between boundaries are highlighted by red squares. Orientation of CTCF binding sites, interactions between CTCF in convergent orientation and schematic of the locus are shown below. $\mathbf{b}, \mathrm{cHi}-\mathrm{C}$ of homozygous Dup. Positions of preserved wild-type interactions between boundaries are indicated by dashed circles. The new contact reflecting the breakpoints of the duplicated region is highlighted by a black arrow. Black arrowheads indicate a strong gain of contacts between the region containing the Fgf8 AER enhancers and the region immediately flanking the centromeric side of the Lbx 1 TAD boundary. The 
rectangular dashed area highlighted by asterisks show increased interactions between the Fgf8 AER enhancers region and the Lbx1 TAD. c, Subtraction of wild-type cHi-C interactions from the Dup mutant. The new contact reflecting the breakpoints of the duplicated region is highlighted by a black arrow. Central and telomeric TAD boundary interactions are not affected (central and telomeric dashed circles), while the centromeric dashed circle highlights a gain of interaction representative of the new extra Lbx1 TAD. Black arrowheads and rectangular dashed area (asterisks) point to the increased interactions involving the Fgf8 AER enhancers region. d, Schematic of Dup configuration. The entire $L b \times 1$ TAD is duplicated and remains unchanged in its content and configuration. The telomeric Fgf8 TAD also remains unchanged. Between the two Lbx1 TADs a neo-TAD forms containing the Fbxw4 gene, the Fgf8 AER enhancers and the small region centromeric of the $L b \times 1$ TAD boundary. Red dashed arrows indicate the ectopic contacts between Fgf8 AER enhancers and the Lbx1 TAD. e, cHi-C of homozygous Inv1. Dashed circles indicate the position of the original wild-type interactions between boundaries, two of them lost upon reshuffling of the boundary between $L b x 1$ and Fgf 8 TADs as a consequence of the inversion. The boundary involved in the inversion is shown as blurry. Dashed lines on the right point out the new smaller Fgf8 TAD, now comprising only Fgf8. Black arrowhead highlights the bow tie configuration representative of the inverted regions. The rectangular dashed area highlighted by asterisks show ectopic interactions compared to wildtype between the Fgf8 AER enhancers region and the Lbx1 TAD. f, Subtraction of wild-type cHi-C interactions from the Inv1 mutant. Centromeric and telomeric dashed circles highlight the loss (blue) of the original interactions between boundaries as a consequence of TAD-reshuffling. Black arrowhead highlights the classic bow tie configuration representative of the inverted regions. The rectangular dashed area highlighted by black asterisks show ectopic interactions compared to wild-type between the Fgf8 AER enhancers region and the $L b \times 1$ TAD, while the square dashed area highlighted by white asterisks points out the loss of interactions between Fgf8 and its AER enhancers. g, Schematic of Inv1 configuration. Upon inversion, the Fgf8 AER enhancers are repositioned within a bigger Lbx1 TAD and can now establish ectopic interactions (red dashed arrows), whereas the Fgf8 TAD is reduced in size due to the inversion of the boundary and contains now only Fgf8, isolated from its AER enhancers. 
a

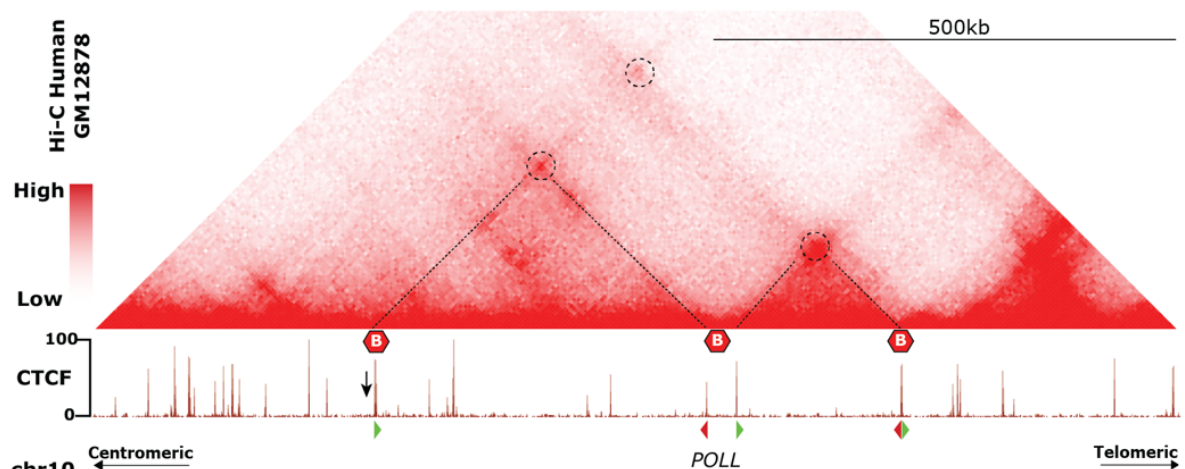

b

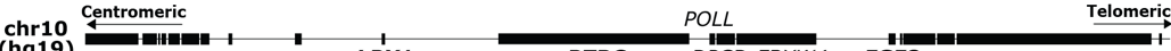

b
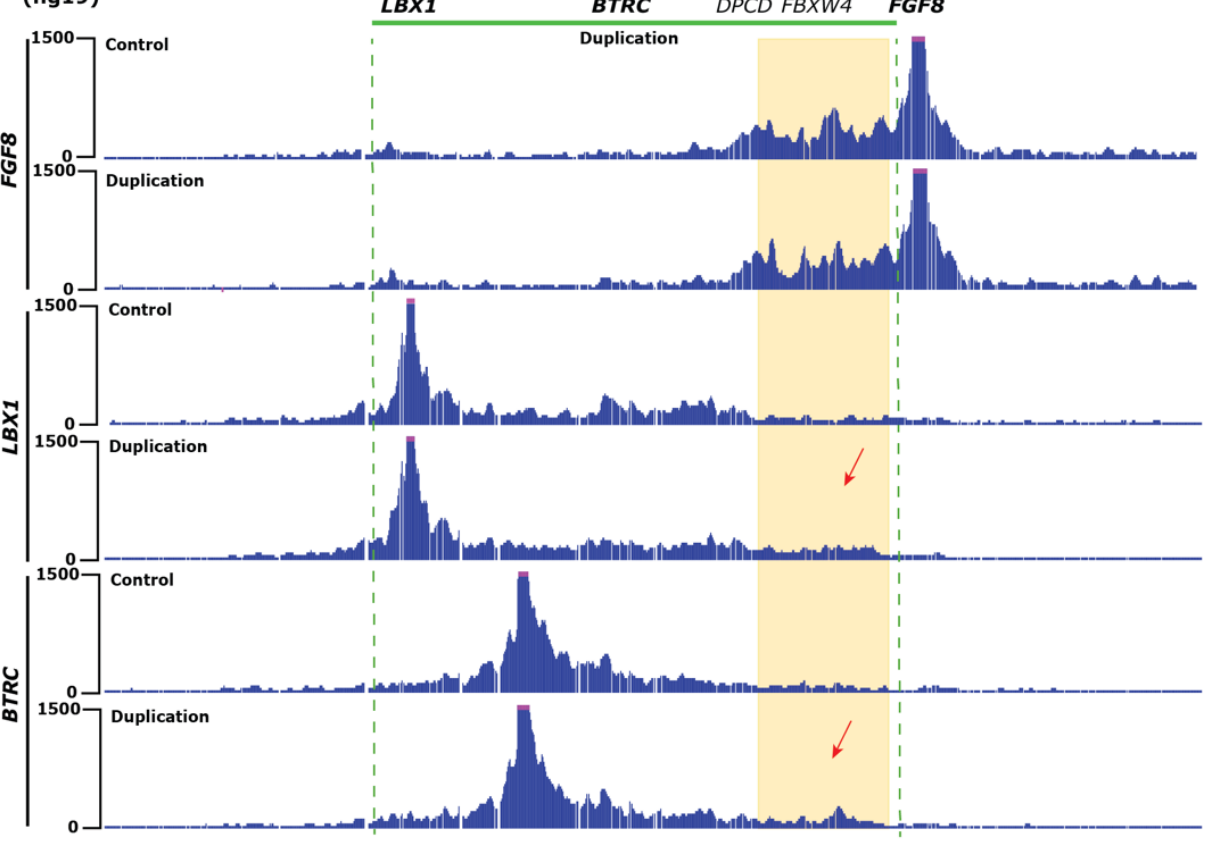

C
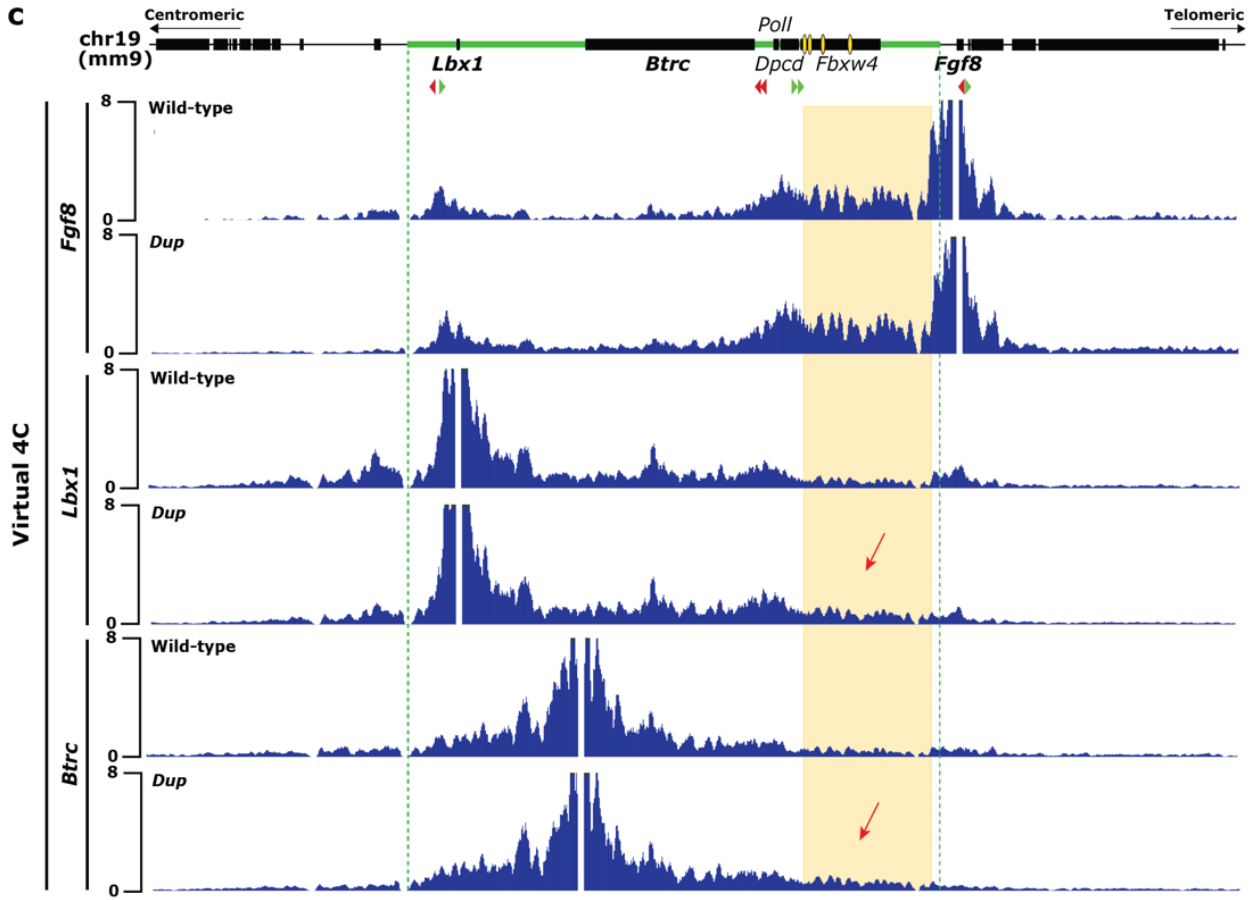
Fig. 3 | 4C-seq in human fibroblasts reveals ectopic interactions involving $L B X 1$ and BTRC, further supported by virtual 4C in mouse. a, Hi-C at the LBX1/FGF8 locus (hg19; chr10:102,669,128-103,840,922) derived from GM12878 cell ${ }^{39}$. TADs structures are similar between human and mouse. CTCF ChIP-seq from GM12878 cells ${ }^{40,41}$, orientation of CTCF binding sites and schematic of the human FGF8 locus are shown below. Black arrow indicates a CTCF binding site in negative orientation at the LBX1 centromeric TAD boundary present in mouse but not in human. b, 4C-seq in human fibroblasts from a healthy control and a patient carrying the SHFM duplication with viewpoints on FGF8, LBX1 and BTRC promoter regions. The yellow area uncovers the region containing the Fgf8 enhancers and the red arrows highlights the gain of ectopic interactions of this region with $L B X 1$ and BTRC upon duplication. Green bar and dashed lines indicate the duplicated region. A second healthy control and SHFM patient were used to validate our findings (data not shown). C, Virtual $4 \mathrm{C}$ plots generated from $\mathrm{cHi}$-c Dup maps with viewpoints on $L b \times 1$, Fgf8 and Btrc promoter regions. The yellow area uncovers the region containing the Fgf8 enhancers (those specific for the AER are indicated as yellow ovals in the schematic of the murine locus) and the red arrows highlights the gain of ectopic interactions of this region with $L b x 1$ and Btrc upon duplication. Green bar and dashed lines indicate the duplicated region. 


\section{a RNA-seq E11.5 limb bud}
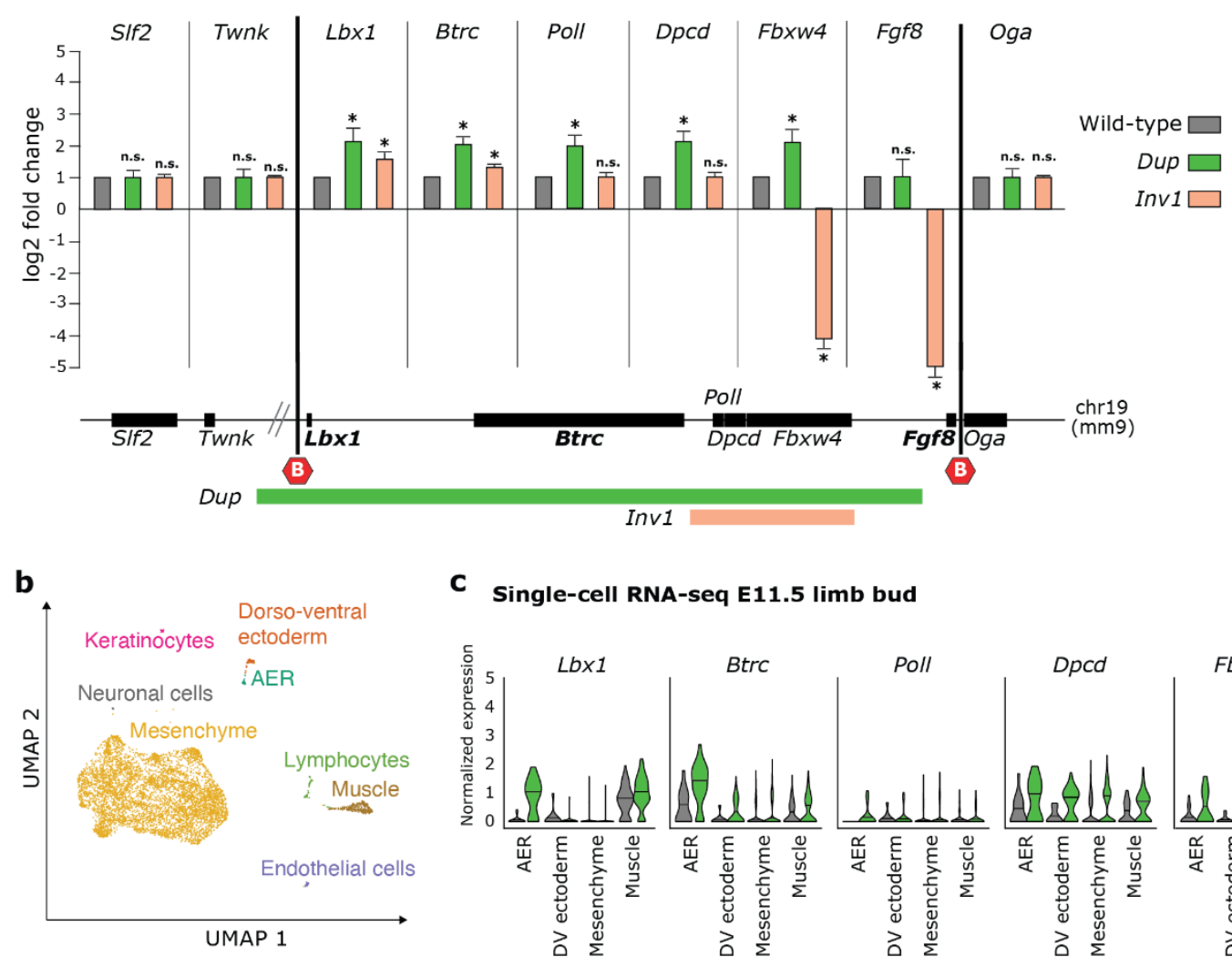

C Single-cell RNA-seq E11.5 limb bud

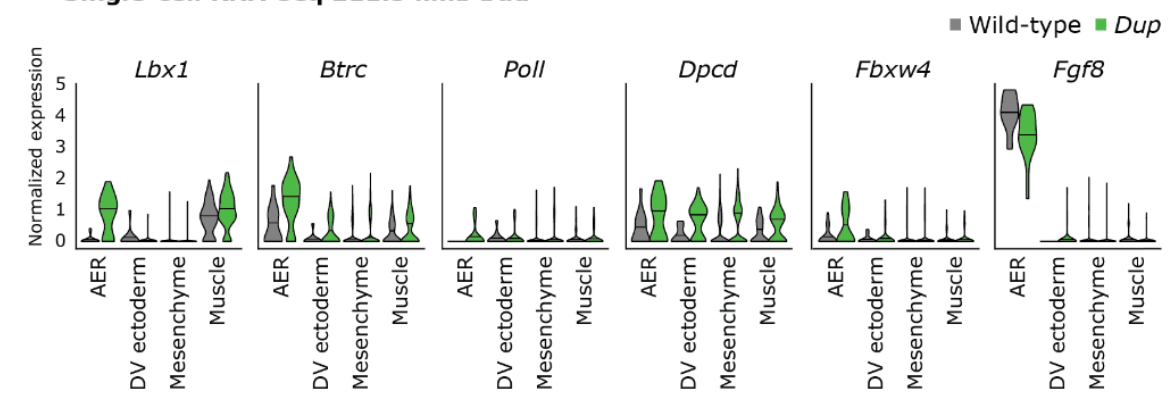

Fig. 4 | Dup and Inv1 chromatin rearrangements have an impact on gene expression. a, Bar plot representing the log2 fold change expression of genes in the Lbx1 and Fgf8 TADs and flanking genes (SIf2 and Twnk (centromeric), Oga (telomeric)) from RNA-seq data of Dup and Inv1 E11.5 limb buds compared to wild-type. Schematic of the locus shows the position of the genes, the TAD boundaries (B) and the parts included in the Dup and the Inv1 mutants. Wild-type Dup and Inv1 samples are shown in black, green and orange, respectively. n.s $=$ non-significant, ${ }^{*}$ Adjusted $p$-value $<0.05$. b, Uniform manifold approximation and projection (UMAP) showing 8 cell clusters identified via scRNA-seq of E11.5 mouse hindlimbs from wild-type and Dup mutant. c, Violin plot representing the normalized expression in AER, dorso-ventral ectoderm, mesenchyme and muscle cells for the 6 genes that are part of the Lbx1 and Fgf8 TADs. Wildtype and Dup samples are shown in black and green respectively. 
a Whole Mount in situ hybridization E11.5

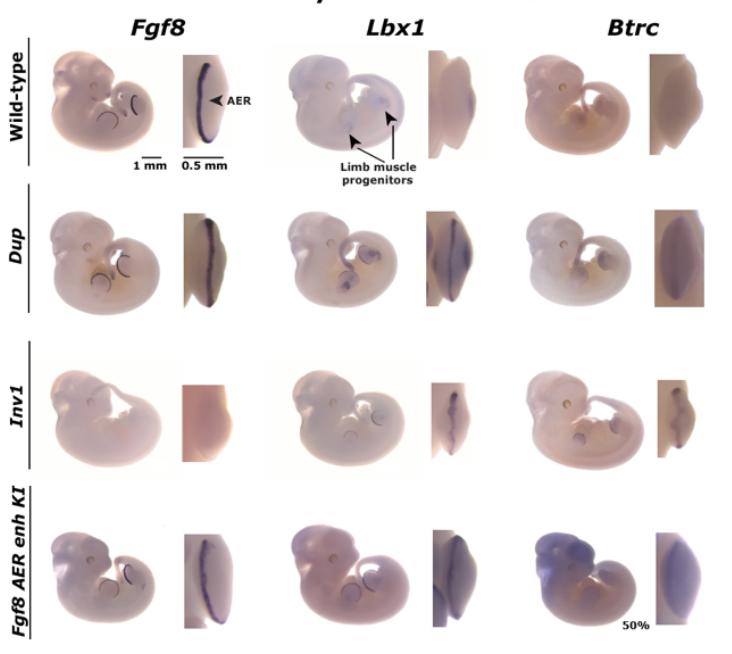

Wild-type
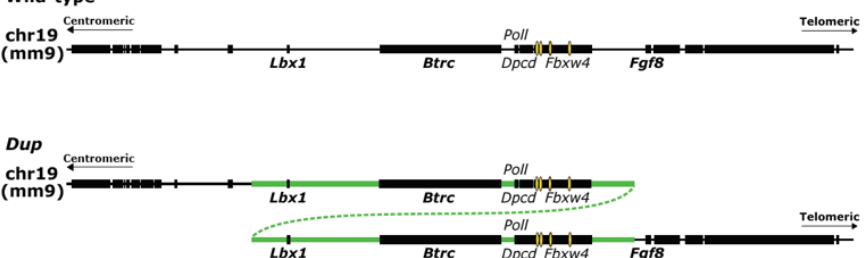

Inv1

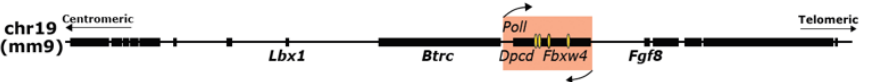

Fgf8 AER enh KI

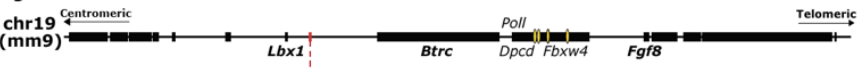

b

Mammalian Phenotype enrichment (top 200 AER up-regulated genes)

C

Myogenic genes expression in AER
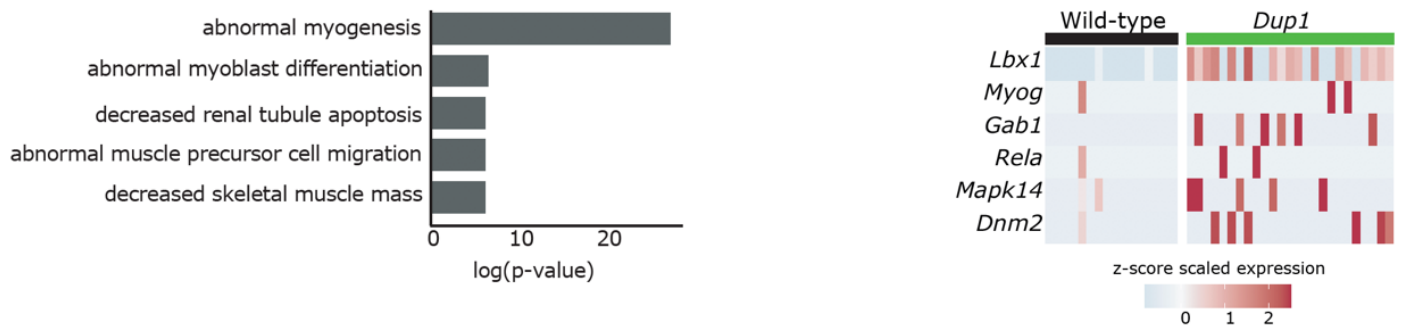

d

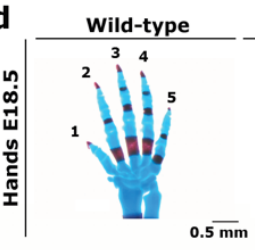

Dup Inv1 Fgf8 AER enh KI
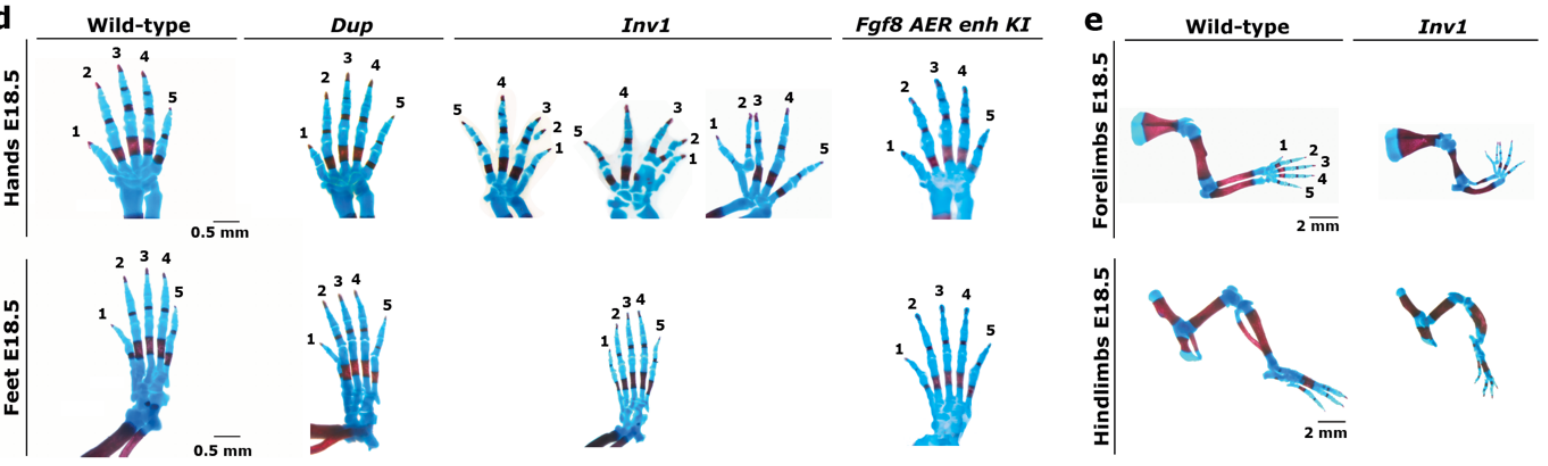

Fig. 5 | Ectopic interactions led to Lbx1 and Btrc misexpression in the AER. a, Whole-mount in situ hybridization for Fgf8, Lbx1 and Btrc at E11.5. Expression was checked and confirmed in at least 3 homozygous embryos (at least $n=3$ biological replicates) (left panel). Schematics of the locus for wild-type and CRISPR/Cas9 alleles (right panel). Yellow ovals highlight Fgf8 AER enhancers. b, GO analysis for the MGI Mammalian phenotype enrichment terms using the top 200 genes that are up-regulated genes in the Dup mutant compared to wild-type in the AER cells from the scRNA-seq data. The 5 most significant enriched terms are represented on a log ( $p$-value) scale. c, Heatmap showing the expression of 6 genes (Lbx1, Myog, Gab1, Rela, Mapk14 and Dnm2) associated with the myogenic enriched terms in (b) in the AER cells. Each column represents one AER cell and the z-score scaled expression is indicated for each gene, showing a global increased expression in the Dup mutant's AER cells. d, Skeletal analysis of E18.5 
limbs stained with alcian blue (cartilage) and alizarin red (bone). Hands and feet from wild-type and homozygous Dup, Inv1 and Fgf8 AER enhancer KI E18.5 embryos. No particular phenotype was observed in feet from all the mutants and in hands from Dup and Fgf8 AER enhancer KI. Fused bones and split digits were instead detected in Inv1 hands. e, Comparison between wild-type and homozygous Inv1 forelimbs and hindlimbs highlight the presence of underdeveloped limbs in Inv1 as a consequence of Fgf8 loss of expression in the AER. 


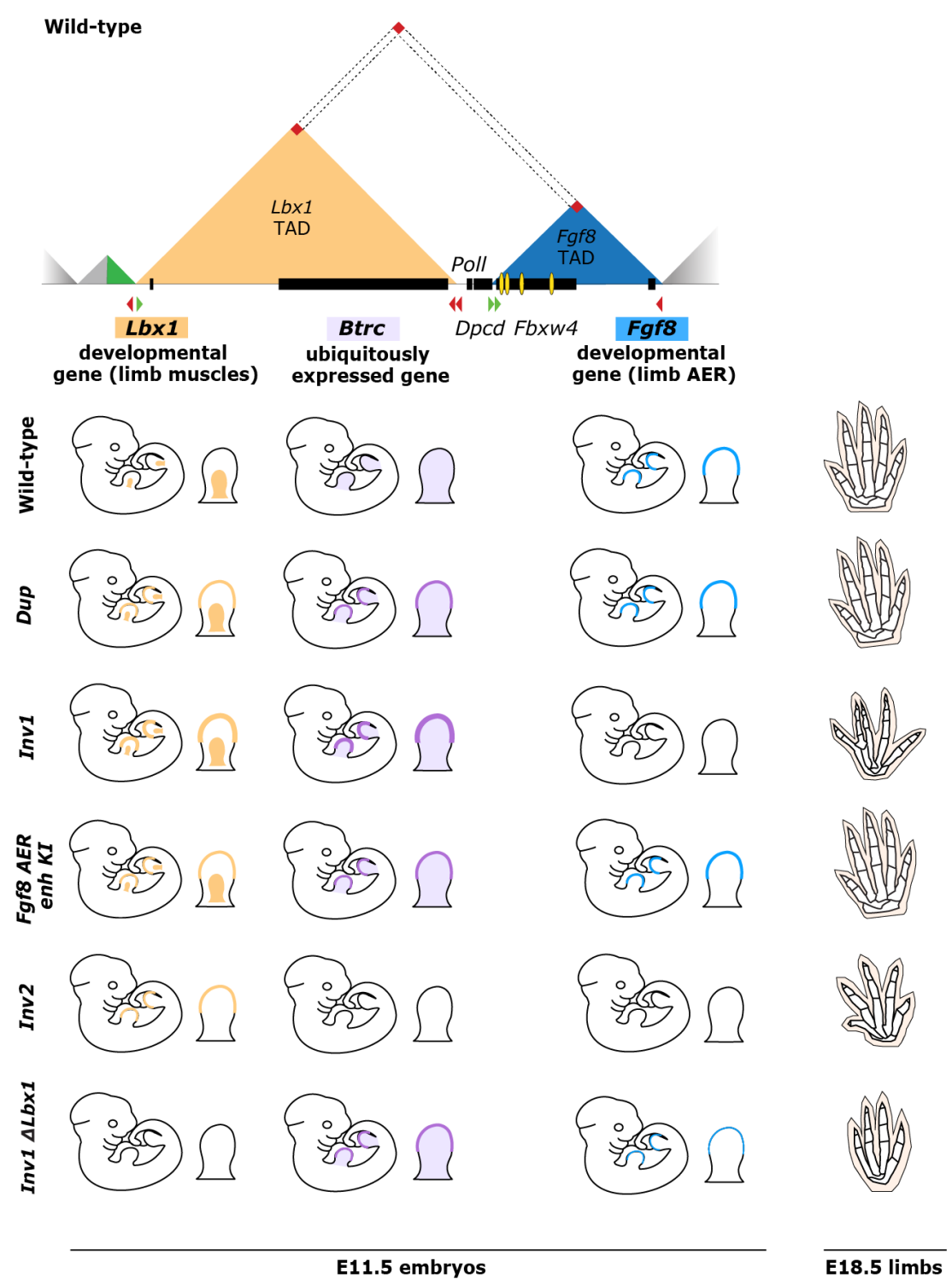

Fig. 6 | Combinatorial effects on gene expression at the Lbx1/Fgf8 locus leads to SHFM3 digit fusion phenotype.

Schematic overview of the Lbx1-Fgf8 TADs (top) and Lbx1, Btrc and Fgf8 expression in the developing limb at E11.5 and skeletal phenotype at E18.5 among the different structural rearrangements (bottom). Yellow and violet patterns represent endogenous expression in the limb bud and misexpression in the AER of $L b \times 1$ and Btrc, respectively. Turquoise pattern highlights Fgf8 expression in the AER. Ectopic expression of Lbx1 and Btrc in the AER is observed for both the Dup and Inv mutant embryos at E11.5. However, only the Inv1 mutants, for which the level of $L b x 1$ and Btrc expression in the AER is higher and Fgf8 expression is missing, show a digit fusion phenotype at E18.5, reminiscent of human SHFM. Ectopic expression of either $L b x 1$ alone (Inv2 mutant) or Btrc alone (Inv1 $L L b x 1)$ together with loss of Fgf8 AER expression is not enough to lead to the digit fusion SHFM-like phenotype but only result in an Fgf8-KO limb phenotype. 


\section{SUPPLEMENTARY FIGURES}

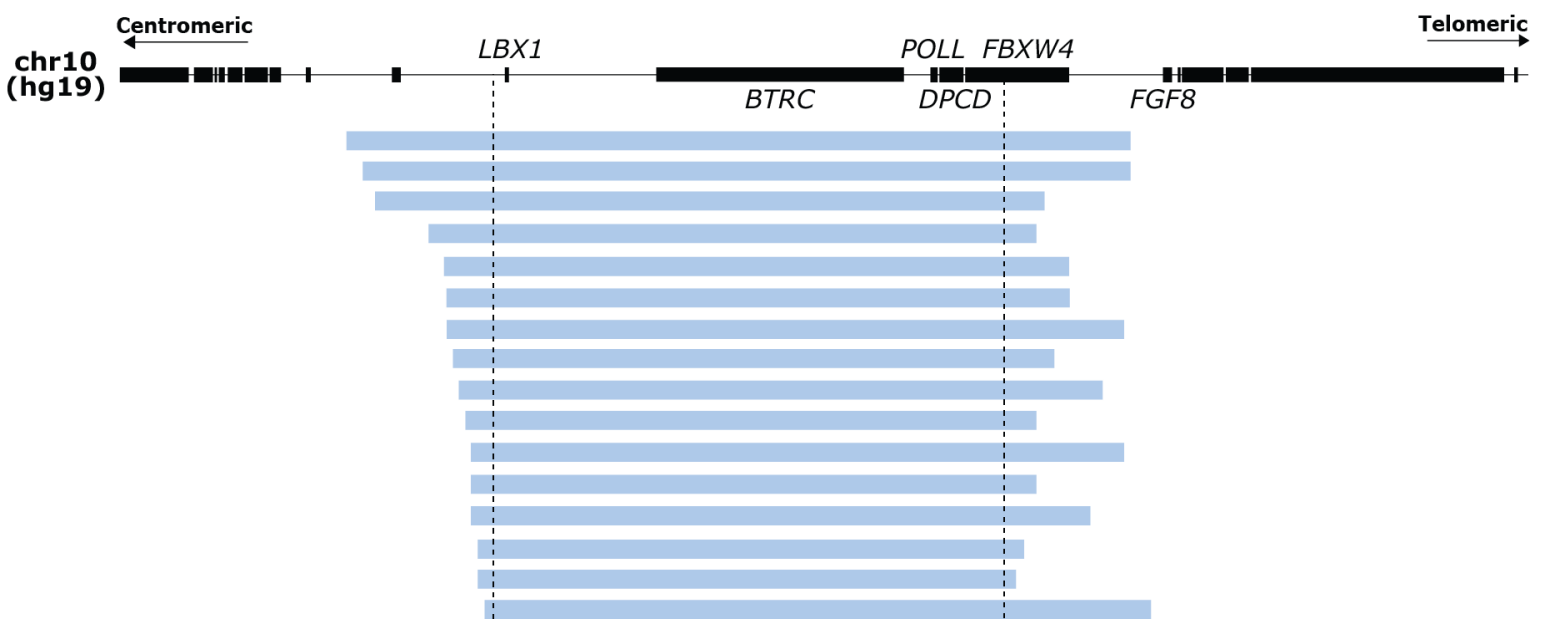

Supplementary Fig. 1 | Overview of in house SHFM3 duplications. All the identified duplications included LBX1 at the centromeric side and excluded FGF8 at the telomeric side. Dashed black lines indicate the minimal critical region of the duplications. 

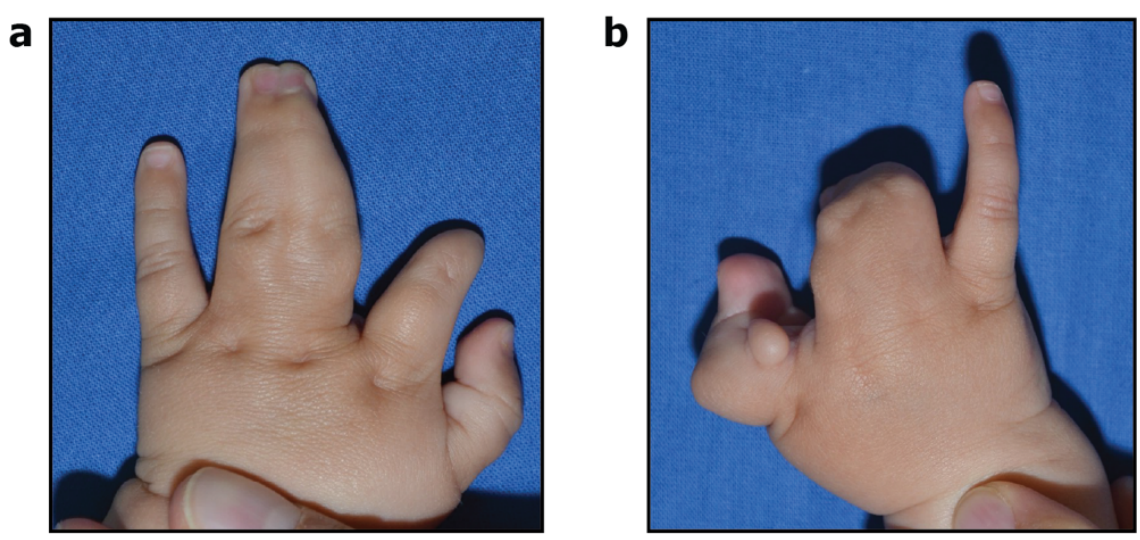

Supplementary Fig. 2 | Patient carrying an inversion at the LBX1/FGF8 locus exhibited two phenotypes of the SHFM spectrum. a, Left hand showing fusion of digit III and IV. b, Right hand showing absence of central distal digits. 
a

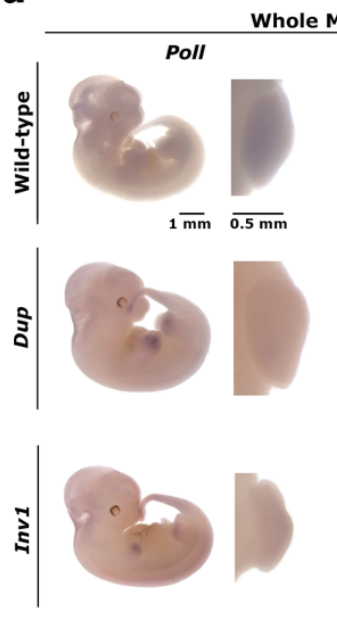

d

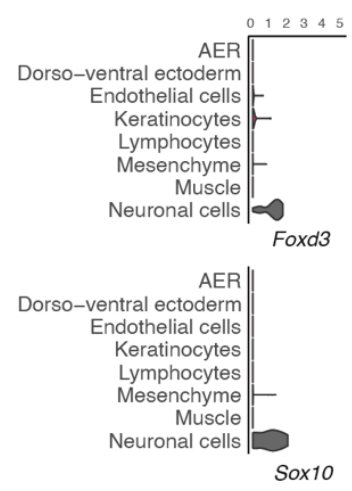

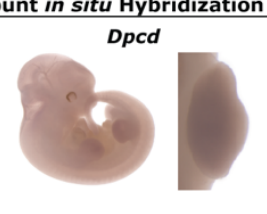
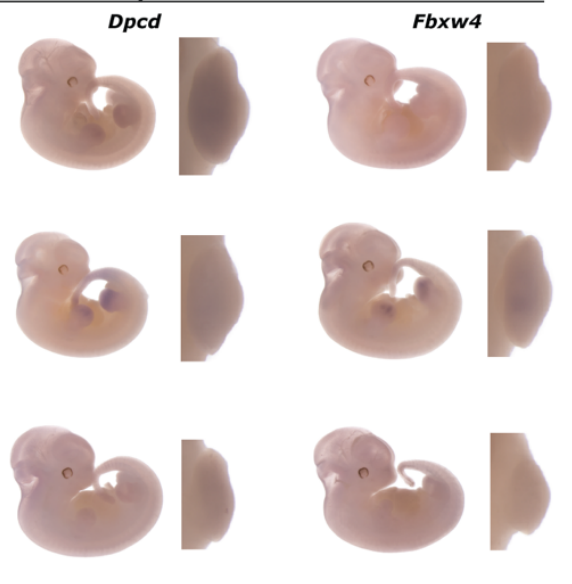

Normalized expression

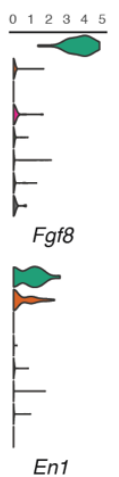

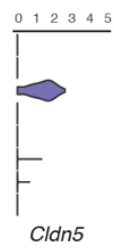

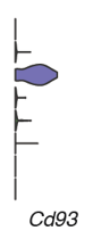

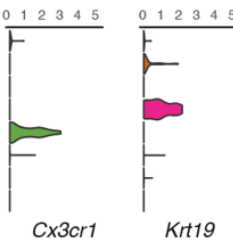
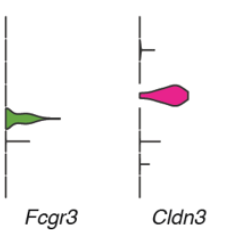

b

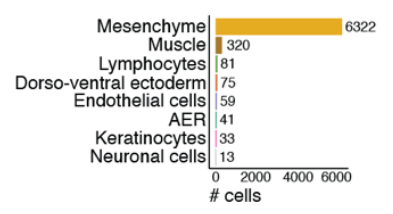

C
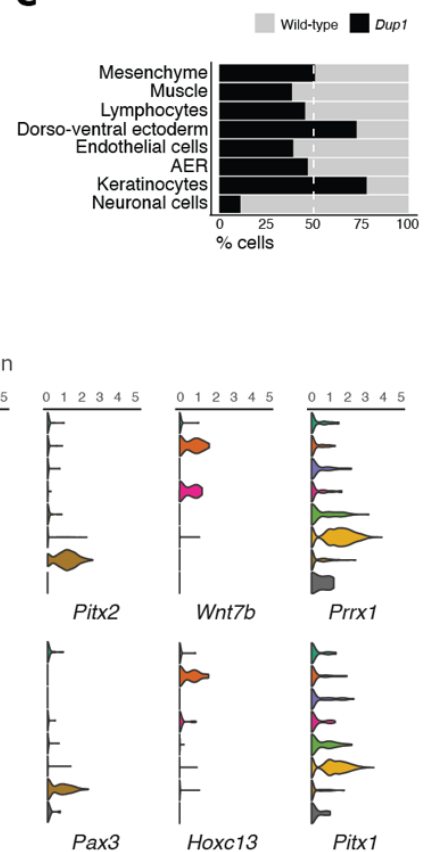

Supplementary Fig. 3 | No misexpression in the AER was detected for other genes at the Lbx1-Fgf8 locus. a, WISH of the other genes at the Lbx1-Fgf8 locus for Dup and Inv1. Expression was checked and confirmed in at least 3 or more homozygous embryos. $\mathbf{b}$, Bar plot representing the number of cells per cluster as defined in Figure $\mathbf{4 b}$. These numbers correspond to the merged data of both wild-type and Dup samples, from the scRNA-seq of E11.5 hindlimbs. c, Bar plot representing the \% of cells per cluster as defined in Figure $\mathbf{4} \mathbf{b}$ for each genotype separately. Some biases are observed, particularly for small cell population, as expected from scRNA-seq data. $\mathbf{d}$, Violin plots representing the expression of two marker genes per cluster that were used to confirm the clustering analysis shown in Figure $\mathbf{4 b}$. By example, AER cells are defined by high expression of Fgf8 and En1. 
a
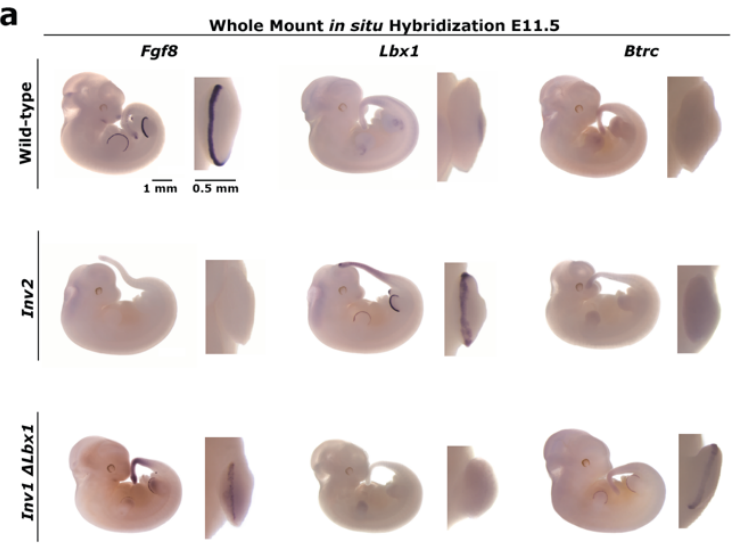

C

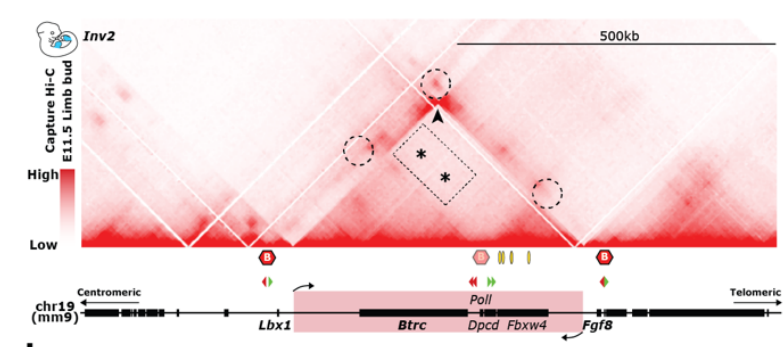

d

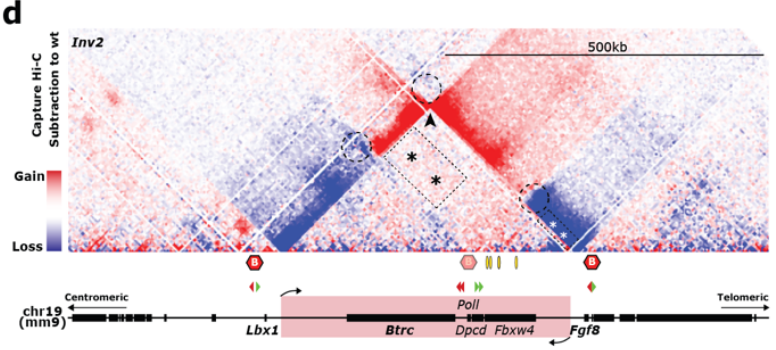

b

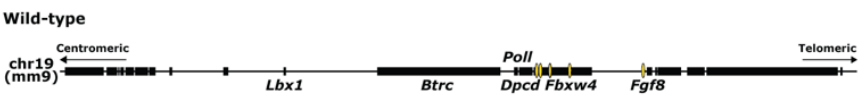

Inv2
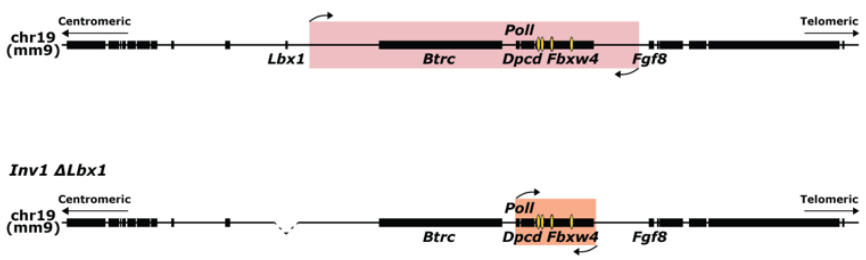

$\mathbf{e}$

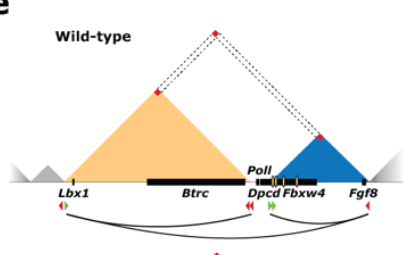

f
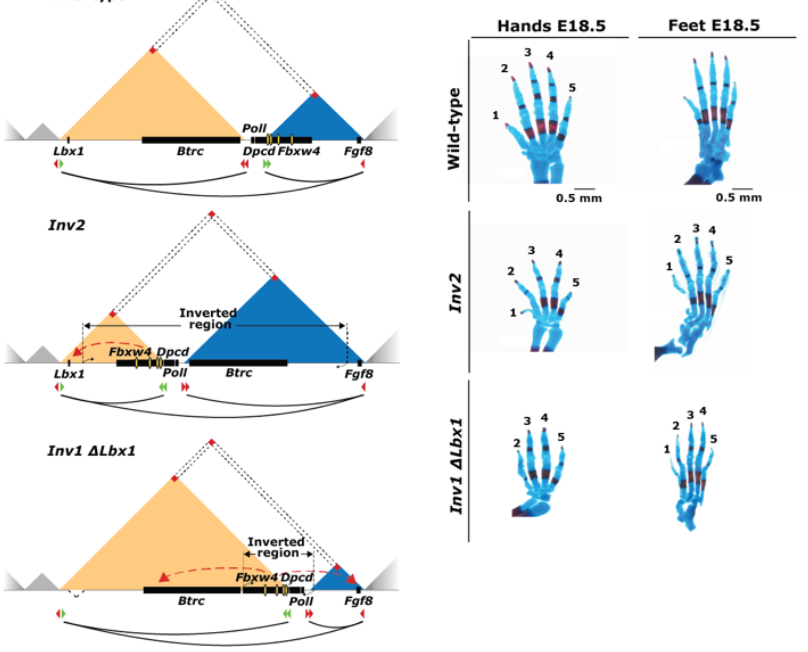

Supplementary Fig. 4 | Misexpression of both $L b \times 1$ and Btrc is potentially required to develop a SHFM phenotype. a, Whole-mount in situ hybridization for Fgf8, Lbx1 and Btrc at E11.5. Expression was checked and confirmed in at least 3 or more homozygous embryos. b, Schematics of the locus for wild-type and CRISPR/Cas9 alleles. Yellow ovals highlight Fgf8 AER enhancers. c, cHi-C of homozygous Inv2. Dashed circles indicate the position of the original wild-type interactions between boundaries, two of them lost upon reshuffling of the boundary between Lbx1 and Fgf8 TADs as a consequence of this inversion. The boundary involved in the inversion is shown as blurry. Black arrowhead highlights the bow tie configuration representative of the inverted regions. The rectangular dashed area highlighted by asterisks show ectopic interactions compared to wild-type between the Fgf8 AER enhancers region and the one containing now only $L b x 1$. d, Subtraction of wild-type $\mathrm{cHi}$-C interactions from Inv2 mutants. Dashed circles highlight the loss (blue) of the original interactions between boundaries as a consequence of TADreshuffling. Black arrowhead highlights the classic bow tie configuration representative of the inverted regions. The rectangular dashed area highlighted by black asterisks show ectopic interactions compared to wild-type between the Fgf8 AER enhancers region and the one containing Lbx1 only, while the rectangular dashed area highlighted by white asterisks points out the loss of interactions between Fgf8 and its AER enhancers. e, (Top) Wild-type schematic. (Middle) Inv2. (Bottom) Inv1 $\Delta L b x 1$. Upon Inv2 the 
Fgf8 regulatory elements are repositioned within a smaller $L b x 1$ TAD without $B t r c$, so that the ectopic interactions are established only with $L b \times 1$ (red dashed arrow), whereas the Fgf8 TAD is increased in size and comprises not only Fgf8, but also Btrc, both isolated from Fgf8 enhancers. f, Skeletal analysis of E18.5 hands and feet stained with alcian blue (cartilage) and alizarin red (bone) from wild-type, Inv2 and Inv1 $\Delta L b \times 1$. 


\section{REFERENCES}

1. Bonev, B. \& Cavalli, G. Organization and function of the 3D genome. Nat. Rev. Genet. 17, 661-678 (2016).

2. Glaser, J. \& Mundlos, S. 3D or Not 3D: Shaping the Genome during Development. Cold Spring Harb. Perspect. Biol. a040188 (2021) doi:10.1101/cshperspect.a040188.

3. Lupiáñez, D. G. et al. Disruptions of Topological Chromatin Domains Cause Pathogenic Rewiring of Gene-Enhancer Interactions. Cell 161, 1012-1025 (2015).

4. Franke, M. et al. Formation of new chromatin domains determines pathogenicity of genomic duplications. Nature 538, 265-269 (2016).

5. Spielmann, M., Lupiáñez, D. G. \& Mundlos, S. Structural variation in the 3D genome. Nat. Rev. Genet. 19, 453-467 (2018).

6. Ghavi-Helm, Y. et al. Highly rearranged chromosomes reveal uncoupling between genome topology and gene expression. Nat. Genet. 51, 1272-1282 (2019).

7. Elliott, A. M. \& Evans, J. A. Genotype-phenotype correlations in mapped split hand foot malformation (SHFM) patients. Am. J. Med. Genet. A. 140A, 1419-1427 (2006).

8. Klopocki, E. et al. Duplications of BHLHA9 are associated with ectrodactyly and tibia hemimelia inherited in non-Mendelian fashion. J. Med. Genet. 49, 119-125 (2012).

9. Tayebi, N. et al. Deletions of exons with regulatory activity at the DYNC1I1 locus are associated with split-hand/split-foot malformation: array CGH screening of 134 unrelated families. Orphanet J. Rare Dis. 9, 108 (2014).

10. Kantaputra, P. N. \& Carlson, B. M. Genetic regulatory pathways of split-hand/foot malformation. Clin. Genet. 95, 132-139 (2019). 
11. Duijf, P. H. G., van Bokhoven, H. \& Brunner, H. G. Pathogenesis of split-hand/split-foot malformation. Hum. Mol. Genet. 12, R51-R60 (2003).

12. Sowińska-Seidler, A., Socha, M. \& Jamsheer, A. Split-hand/foot malformation molecular cause and implications in genetic counseling. J. Appl. Genet. 55, 105-115 (2014).

13. Holder-Espinasse, M. et al. Duplication of 10q24 locus: broadening the clinical and radiological spectrum. Eur. J. Hum. Genet. EJHG 27, 525-534 (2019).

14. de Mollerat, X. J. et al. A genomic rearrangement resulting in a tandem duplication is associated with split hand-split foot malformation 3 (SHFM3) at 10q24. Hum. Mol. Genet. 12, 1959-1971 (2003).

15. Buckingham, M. Skeletal muscle formation in vertebrates. Curr. Opin. Genet. Dev. 11, 440-448 (2001).

16. Niswander, L. Interplay between the molecular signals that control vertebrate limb development. Int. J. Dev. Biol. 46, 877-881 (2002).

17. Busino, L. et al. Degradation of Cdc25A by $\beta$-TrCP during $S$ phase and in response to DNA damage. Nature 426, 87-91 (2003).

18. Zariwala, M. et al. Investigation of the possible role of a novel gene, DPCD, in primary ciliary dyskinesia. Am. J. Respir. Cell Mol. Biol. 30, 428-434 (2004).

19. García-Díaz, M. et al. DNA polymerase lambda (Pol $\lambda$ ), a novel eukaryotic DNA polymerase with a potential role in meiosis11Edited by M. Yaniv. J. Mol. Biol. 301, 851-867 (2000).

20. Lockwood, W. W., Chandel, S. K., Stewart, G. L., Erdjument-Bromage, H. \& Beverly, L. J. The Novel Ubiquitin Ligase Complex, SCFFbxw4, Interacts with the COP9 Signalosome in an F- 
Box Dependent Manner, Is Mutated, Lost and Under-Expressed in Human Cancers. PLoS One 8, e63610 (2013).

21. Kraft, K. et al. Deletions, Inversions, Duplications: Engineering of Structural Variants using CRISPR/Cas in Mice. Cell Rep. 10, 833-839 (2015).

22. Fudenberg, G. et al. Formation of Chromosomal Domains by Loop Extrusion. Cell Rep. 15, 2038-2049 (2016).

23. Gross, M. K. et al. Lbx1 is required for muscle precursor migration along a lateral pathway into the limb. Dev. Camb. Engl. 127, 413-424 (2000).

24. Moon, A. M. \& Capecchi, M. R. Fgf8 is required for outgrowth and patterning of the limbs. Nat. Genet. 26, 455-459 (2000).

25. Marinić, M., Aktas, T., Ruf, S. \& Spitz, F. An integrated holo-enhancer unit defines tissue and gene specificity of the Fgf8 regulatory landscape. Dev. Cell 24, 530-542 (2013).

26. Hörnblad, A., Bastide, S., Langenfeld, K., Langa, F. \& Spitz, F. Dissection of the Fgf8 regulatory landscape by in vivo CRISPR-editing reveals extensive intra- and inter-enhancer redundancy. Nat. Commun. 12, 439 (2021).

27. Nait-Oumesmar, B. et al. Ectopic expression of $\mathrm{Gcm} 1$ induces congenital spinal cord abnormalities. Dev. Camb. Engl. 129, 3957-3964 (2002).

28. Zaffran, S., Odelin, G., Stefanovic, S., Lescroart, F. \& Etchevers, H. C. Ectopic expression of Hoxb1 induces cardiac and craniofacial malformations. Genes. N. Y. N 2000 56, e23221 (2018). 
29. Balling, R., Mutter, G., Gruss, P. \& Kessel, M. Craniofacial abnormalities induced by ectopic expression of the homeobox gene Hox-1.1 in transgenic mice. Cell 58, 337-347 (1989).

30. Kano, H. et al. Genomic rearrangement at 10q24 in non-syndromic split-hand/split-foot malformation. Hum. Genet. 118, 477-483 (2005).

31. Lewandoski, M., Sun, X. \& Martin, G. R. Fgf8 signalling from the AER is essential for normal limb development. Nat. Genet. 26, 460-463 (2000).

32. Gurrieri, F. \& Everman, D. B. Clinical, genetic, and molecular aspects of split-hand/foot malformation: an update. Am. J. Med. Genet. A. 161A, 2860-2872 (2013).

33. Despang, A. et al. Functional dissection of the Sox9-Kcnj2 locus identifies nonessential and instructive roles of TAD architecture. Nat. Genet. 51, 1263-1271 (2019).

34. Friedli, M. et al. Characterization of mouse Dactylaplasia mutations: a model for human ectrodactyly SHFM3. Mamm. Genome Off. J. Int. Mamm. Genome Soc. 19, 272-278 (2008).

35. Zuniga, A. \& Zeller, R. Dynamic and self-regulatory interactions among gene regulatory networks control vertebrate limb bud morphogenesis. Curr. Top. Dev. Biol. 139, 61-88 (2020).

36. Verheyden, J. M. \& Sun, X. Embryology meets molecular biology: Deciphering the apical ectodermal ridge. Dev. Biol. 429, 387-390 (2017).

37. Demetrius, L. Of mice and men. EMBO Rep. 6, S39-S44 (2005).

38. Allou, L. et al. Non-coding deletions identify Maenli IncRNA as a limb-specific En1 regulator. Nature 592, 93-98 (2021). 
39. Rao, S. S. P. et al. A 3D Map of the Human Genome at Kilobase Resolution Reveals

Principles of Chromatin Looping. Cell 159, 1665-1680 (2014).

40. Dunham, l. et al. An integrated encyclopedia of DNA elements in the human genome.

Nature 489, 57-74 (2012).

41. Davis, C. A. et al. The Encyclopedia of DNA elements (ENCODE): data portal update.

Nucleic Acids Res. 46, D794-D801 (2018).

42. Artus, J. \& Hadjantonakis, A.-K. Generation of chimeras by aggregation of embryonic stem cells with diploid or tetraploid mouse embryos. Methods Mol. Biol. Clifton NJ 693, 3756 (2011).

43. Knight, P. \& Ruiz, D. A fast algorithm for matrix balancing. IMA J. Numer. Anal. 33, 10291047 (2013).

44. Dobin, A. et al. STAR: ultrafast universal RNA-seq aligner. Bioinformatics 29, 15-21 (2013).

45. Love, M. I., Huber, W. \& Anders, S. Moderated estimation of fold change and dispersion for RNA-seq data with DESeq2. Genome Biol. 15, 550 (2014).

46. Wolock, S. L., Lopez, R. \& Klein, A. M. Scrublet: Computational Identification of Cell Doublets in Single-Cell Transcriptomic Data. Cell Syst. 8, 281-291.e9 (2019).

47. Hafemeister, C. \& Satija, R. Normalization and variance stabilization of single-cell RNAseq data using regularized negative binomial regression. Genome Biol. 20, 296 (2019).

48. Butler, A., Hoffman, P., Smibert, P., Papalexi, E. \& Satija, R. Integrating single-cell transcriptomic data across different conditions, technologies, and species. Nat. Biotechnol. 36, 411-420 (2018). 
49. Cao, J. et al. The single-cell transcriptional landscape of mammalian organogenesis.

Nature 566, 496-502 (2019).

50. Chen, E. Y. et al. Enrichr: interactive and collaborative HTML5 gene list enrichment analysis tool. BMC Bioinformatics 14, 128 (2013).

51. Krijger, P. H. L., Geeven, G., Bianchi, V., Hilvering, C. R. E. \& de Laat, W. 4C-seq from beginning to end: A detailed protocol for sample preparation and data analysis. Methods San Diego Calif 170, 17-32 (2020). 